Article

\title{
Large-Eddy Simulations on the Effects of Two Wind Passage Types between Buildings on the Airflow and Drag Characteristics
}

\author{
Lu Wang ${ }^{1}$, Jing Liu ${ }^{2, *}$, Cunyan Jiang ${ }^{1,2}$, Biao Li $^{3}$, Di Song ${ }^{1}\left({ }\right.$, Ming Lu ${ }^{1,2}$ and Yingli Xuan ${ }^{4}$ \\ 1 School of Architecture, Harbin Institute of Technology, Harbin 150001, China; wanglu_annie@163.com (L.W.); \\ hit_jcy@163.com (C.J.); 18b934024@stu.hit.edu.cn (D.S.); hitlm@126.com (M.L.) \\ 2 Key Laboratory of Cold Region Urban and Rural Human Settlement Environment Science and Technology, \\ Ministry of Industry and Information Technology, Beijing 100804, China \\ 3 School of Energy Science and Engineering, Harbin Institute of Technology, Harbin 150001, China; \\ biaoli@hit.edu.cn \\ 4 Department of Architecture, Tokyo Polytechnic University, Tokyo 164-8678, Japan; y.xuan@arch.t-kougei.ac.jp \\ * Correspondence: liujinghit0@163.com
}

Citation: Wang, L.; Liu, J.; Jiang, C.; Li, B.; Song, D.; Lu, M.; Xuan, Y. Large-Eddy Simulations on the Effects of Two Wind Passage Types between Buildings on the Airflow and Drag Characteristics. Atmosphere 2021, 12, 1646. https://doi.org/ 10.3390/atmos 12121646

Academic Editor: Zhaoxia Pu

Received: 22 October 2021

Accepted: 3 December 2021

Published: 8 December 2021

Publisher's Note: MDPI stays neutral with regard to jurisdictional claims in published maps and institutional affiliations.

Copyright: (c) 2021 by the authors. Licensee MDPI, Basel, Switzerland. This article is an open access article distributed under the terms and conditions of the Creative Commons Attribution (CC BY) license (https:// creativecommons.org/licenses/by/ $4.0 /)$.

\begin{abstract}
Passages between buildings comprise the airflow path through the buildings, and the wind passage is often studied in terms of two buildings located parallel or at a certain angle. From the perspective of urban areas, the wind passage can be considered the series connection of all local wind passages between each row of buildings. Whether the central axis of each local wind passage is collinear or not, the wind passages of the building array can be summarized as distorted and streamlined types. Large-eddy simulations (LESs) are employed to assess the impacts of the above two wind passage types on the airflow and drag characteristics. The mean, unsteady flow fields and the drag distributions are discussed to assess the effects of wind passages types. Span-wise airflow was found in the wake region in the case of distorted wind passages (DWP), whereas the recirculating vortices dominated the wake region for the case of streamlined wind passages (SWP). Span-wise airflow enhanced the mean stream-wise velocity $U$ and span-wise velocity $U$ in the wake region, decreased $U$ in the wind passage region, and increased dispersive stress $\left\langle\widetilde{V}^{2}\right\rangle$ and $\left\langle\widetilde{U}^{2}\right\rangle$ within the urban canopy and the peak Reynolds stress above the urban canopy. Further, it strengthened the individual drag forces of buildings and the fluctuations of span-wise and stream-wise individual drag forces. The air of DWP penetrated deeper than SWP. These findings provide theory and data support for better design of wind passages between buildings and may serve as a foundation for urban design and planning.
\end{abstract}

Keywords: large-eddy simulation; wind passage; drag force distributions; flow characteristics

\section{Introduction}

Urbanization is the progress of human society and the crystallization of human civilization. The urbanization of modern cities has introduced many environmental issues, such as the heat island effect [1-3], weak ventilation [4-6], and air pollution [7,8].

A wind passage refers to the airflow path between buildings along the wind direction. The wind conditions in the passages can reflect the interference effects of the buildings, which obstruct and drag the approaching wind, thereby resulting in the attenuation of the atmospheric flow. Blocken et al. [9] investigated the wind conditions for the passages between two parallel buildings. They found three governing flow regimes, resistance flow, interaction flow and isolated flow, which depend on the passage width and building influence scale. Wind speed in the passage did not show an obvious increase except for the ground level. Blocken et al. [10] further studied the wind passage between two non-parallel buildings and showed that the wind speed amplification factors in diverging passages are generally larger than in converging passages. Li et al. [11] expanded the setup for 
the building orientation of Blocken et al. [10] to a wider range and concluded that the combination of corner streams is the primary determiner of passage flow. Tse et al. [12] explored the impact of twisted wind (wind direction varying with height altitude) on the two wind passages between three consecutive parallel buildings. They concluded that the wind conditions are asymmetric, and the wind velocities are dissimilar for the two passages. Allegrini et al. [13] investigated cases with various orientations of two buildings by wind tunnel experiments. They discovered that from the ground to the roof level, the wind speeds in the passage increase and decrease with angles for the converging and diverging cases, respectively. Jamińska-Gadomska et al. [14] studied wind conditions between two angled buildings based on the actual full-scale structure of a church bell tower. They reported that the wind velocity behind the passage of diverging walls shows the biggest amplification, whereas the wind velocity in front of the contraction of converging walls shows the largest reduction. Yoshida et al. [15] conducted LESs to investigate the effects of height variability on turbulent flows. In their research, two experiments were carried out, one using actual buildings in Kyoto, Japan, and one using buildings with the same heights and equal to the mean building height of the Kyoto buildings. They found that $\lambda_{\mathrm{p}}$ of approximately 0.3 is the threshold to distinguish the prominent effect of height variability. Antoniou et al. [16] investigated outdoor ventilation using air delay by wind tunnel experiment and CFD model. They assessed the ventilation conditions in a dense district in Nicosia, Cyprus. It was found that the height variability of buildings can decrease air delay, which is negatively correlated with the breathability in complex urban areas.

Urban geometry can shape the wind passages to some extent, and there is abundant research regarding the effect of urban geometry on airflow. Cheng et al. [17] conducted wind tunnel experiments to investigate the effects of two layouts (aligned and staggered) of idealized buildings on aerodynamic characteristics. The results showed that the drag coefficient of staggered arranged buildings is larger than that of aligned buildings, which was found by the DNS simulation results of Coceal et al. [18]. Hagishima et al. [19] investigated the effects of layouts (square, staggered and diamond-shaped) by wind tunnel experiments and concluded that the diamond layout behaves the largest drag force. Zaki et al. [20] measured pressure drag, total drag, and wind profile to study urban wind-induced ventilation, considering rectangular block arrays with different packing densities in staggered, square, and diamond layouts. They reported that the drag coefficient of diamond, staggered, and square arrays are in descending order. Hu et al. [21] discovered that the ventilation efficiency of buildings with staggered layout is better than that of buildings with square layout in terms of velocity ratio. Lin et al. [22] found that buildings with square layout have better ventilation efficiency considering the purge flow rate (PFR) using the RANS model. Chen et al. [23] carried out unsteady RANS simulations to investigate the impacts of staggered and square layouts on outdoor ventilation. They concluded that mean velocity ratios at the pedestrian level, and the air change rates of an entire canyon are larger for staggered than square arrays, but the air change rates at roof level for the square array exceed the staggered array. Hanna et al. [24] compared LES results with experimental data and found higher velocities between buildings in the square array than in the staggered arrays. Kono et al. [25] performed LES of the airflow around the square and staggered arrays with various plan area densities, and results confirmed that the spatial mean velocities and sectional drag coefficient are more sensitive to planar area density $\lambda_{p}$ for the staggered case than the square case.

There are three limitations of the above-mentioned research: (1) The research objectives related to the effect of urban geometry are idealized building arrays, where the buildings are evenly distributed. However, the idealized building arrays oversimplify the actual urban buildings, excluding the spatial heterogeneity representatives. (2) For idealized arrays, the flow field around each building and the drag force of individual buildings are identical in the idealized arrays, unlike those in real cities, so there is a need for an in-depth study of the flow field and drag distribution for heterogeneous building arrays. 
(3) Studies concerning wind passages focus on the wind conditions of two buildings located parallel or at a certain angle, lacking the investigation of the wind passages for the total building array. For the building array, wind passages are the series connection of all local wind passages between two rows of buildings. Based on the above analysis and taking into account that wind passages can feature the spatial heterogeneity of building arrays, we summarized two typical types of wind passages for building complexes in real cities (Figure 1), the distorted and streamlined wind passages, according to whether the central axis of each local wind passage (LWP) was collinear or not, as shown in Figure 2. Figure 1 is the array with distorted wind passages, and Figure 2 is the array with streamlined wind passages. The central axes of LWP for distorted wind passages are not collinear. The central axes of LWP for streamlined wind passages are collinear. The main objective of this work is to evaluate the effects of two typical wind passages on the flow characteristics and the drag force distributions. Section 2 illustrates the inflow generation method and the numerical setup for LESs. Section 3 presents the case configuration and the reference wind tunnel experiments, and then validates the LES results with the wind tunnel data. Section 4 discusses the effects of wind passages on the flow characteristics and drag forces of building models. Section 5 summarizes the findings of the present research. This study can help to increase understanding of the influence of different wind passage types on ventilation characteristics to provide necessary data support for improving local climate with proper urban planning and architectural design.

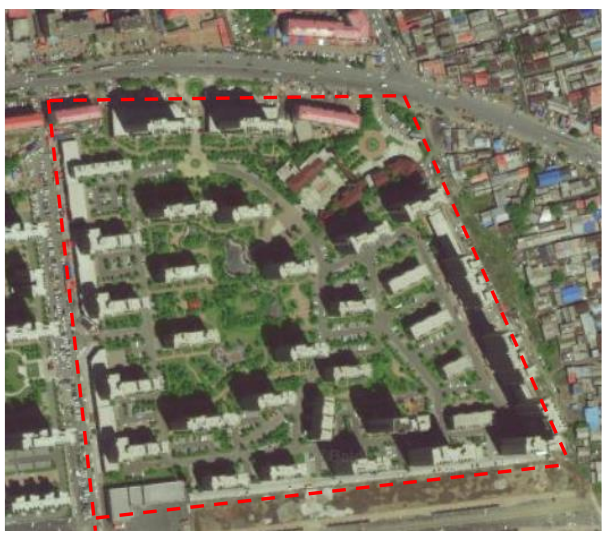

(a)

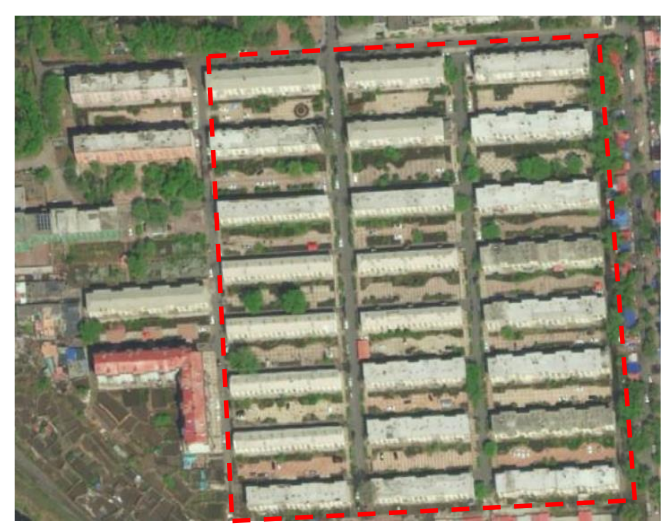

(b)

Figure 1. Actual building layouts in Harbin, China, with (a) distorted wind passages and (b) streamlined wind passages.

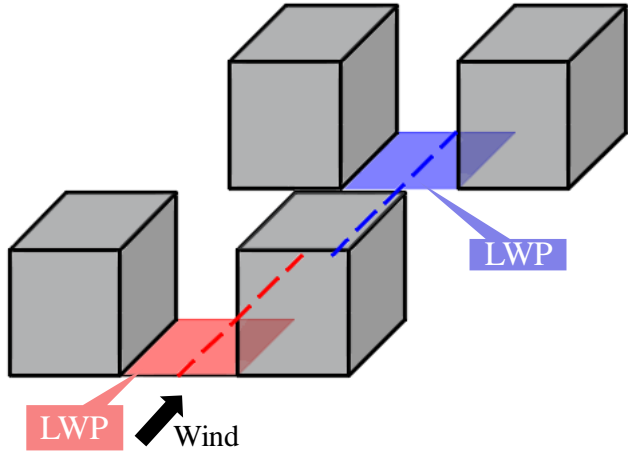

(a)

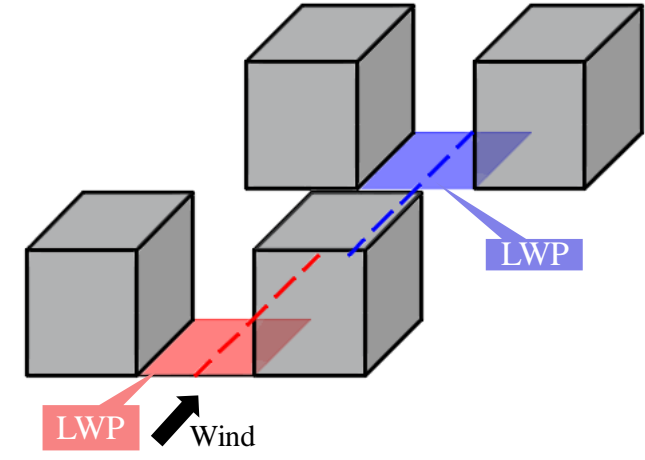

(b)

Figure 2. The location diagram of the two typical wind passages of a building array. (a) Distorted wind passage. (b) Streamlined wind passage. The local wind passages between two buildings are named LWP. 


\section{LES Methodologies}

\subsection{Numerical Setup}

The Parallelized Large-Eddy Simulation Model (PALM) [26], an open-source code developed by Leibniz University Hannover, was used in this study. PALM has been widely applied in many studies, such as the turbulent airflow in a street canyon [8], the turbulent structure of an urban boundary layer [27,28], the urban wind environment [29-32], and fields such as pollutant dispersion [8,33,34]. PALM adopts the LES turbulence model, in which eddies smaller than cut-off length are modeled while large eddies are directly resolved. The calculation grid and discrete operation are used as implicit filters for the Navier-Stokes (NS) equation, and then the turbulence field is divided into the resolved scale and sub-grid scale. The filtered $N$-S equations are as follows:

$$
\begin{gathered}
\frac{\partial \hat{u}_{i}}{\partial x_{i}}=0 \\
\frac{\partial \hat{u}_{i}}{\partial t}+\overline{u_{j}} \frac{\partial \hat{u}_{i}}{\partial x_{j}}=-\frac{1}{\rho} \frac{\partial \hat{p}}{\partial x_{j}}-\frac{\partial \tau_{i j}}{\partial x_{j}}
\end{gathered}
$$

where $u_{\mathrm{i}}$ and $u_{\mathrm{j}}$ are the velocity components and $\rho$ is density. The^symbol denotes the filtering operator, and $\tau_{\mathrm{ij}}$ is the sub-grid stress (SGS) term:

$$
\tau_{i j}=u_{i} \hat{u}_{j}-\hat{u}_{i} \hat{u}_{j}
$$

Based on the Boussinesq turbulent viscosity hypothesis, a modified version of Deardorff's subgrid-scale model [35] was adopted here to calculate the SGS stress term, as follows:

$$
\begin{gathered}
\tau_{i j}-\frac{2}{3} e \delta_{i j}=-v_{S G S}\left(\frac{\partial \hat{u}_{i}}{\partial x_{j}}+\frac{\partial \hat{u}_{j}}{\partial x_{i}}\right) \\
v_{S G S}=C_{m} l e^{\frac{1}{2}}
\end{gathered}
$$

where $v_{\mathrm{SGS}}$ is the SGS turbulent viscosity, $e$ is the SGS turbulent kinetic energy (TKE), $C_{\mathrm{m}}$ of 0.1 is the model constant, and $l$ is the SGS mixing length. For the neutral boundary layer, $l$ depends on the altitude from the wall $z$ and grid spacing. It is calculated as follows:

$$
l=\min \left(1.8 z,(\Delta x \Delta y \Delta z)^{\frac{1}{3}}\right)
$$

The model closure includes a prognostic equation for the SGS-TKE $e$. Under the neutral boundary layer, it is calculated as follows:

$$
\frac{\partial e}{\partial t}=-\hat{u}_{j} \frac{\partial e}{\partial x_{j}}-\tau_{i j}\left(\frac{\partial \hat{u}_{i}}{\partial x_{j}}\right)+\frac{\partial}{\partial x_{j}}\left(2 v_{S G S} \frac{\partial e}{\partial x_{i}}\right)-\epsilon
$$

where $\epsilon$ is the dissipation rate within a grid volume:

$$
\epsilon=C_{\epsilon} \frac{e^{\frac{1}{2}}}{l}
$$

The coefficient $C_{\epsilon}$ was specified as 0.93 . All variables normal to the outflow boundary were set as zero gradients to reduce the impact of the outflow on the inner flow state [36]. Periodic and free slip boundary conditions were used as the span-wise and the top boundary conditions. Wall functions were based on Monin-Obukhov similarity theory [37], so the SGS of the first vertical layer $\tau_{i 3}$ can be calculated as follows:

$$
\tau_{i 3}=-\left[\frac{\hat{u_{r}}}{\log \left(z_{s} / z_{0}\right)}\right]^{2} \frac{\hat{u}_{i}}{\hat{u}_{r}}
$$


where $z_{0}$ is aerodynamic roughness length; $\kappa$ is von Karman constant, set as $0.4 ; z_{\mathrm{s}}$ is the altitude at the first vertical grid; and $u_{r}=\left(u^{2}+v^{2}\right)^{1 / 2}$ is the resolved resultant horizontal velocity at the first vertical grid.

The advection term of the N-S equation adapted the fifth order upwind scheme of Wicker and Skamarock. The time-step scheme us $\mid \mathrm{d}$ for integrating the prognostic variables was the third order of the Runge-Kutta scheme. The Poisson solver for pressure used the multigrid method [38]. The physical time step, $\Delta t$, was dynamically adjusted to meet the criterion that the $C F L$ number was no more than unity. The simulation time was $40 \mathrm{~s}$. For the first $10 \mathrm{~s}$, the flow reached a quasi-steady state. Sliding averages of $10 \mathrm{~s}$ of data among the remaining $30 \mathrm{~s}$ for mean velocity and turbulence intensity profiles showed insignificant changes, so averages of $10 \mathrm{~s}$ was used to perform time averaging for the LES results.

\subsection{Inflow Turbulence Generation Method}

Large-eddy simulation (LES) has been extensively used to study flow over building arrays immersed in boundary layers. This research adopts an internal mapping method [39], which is an implementation of the recycling method modified by Kataoka and Mizuno [40], to generate turbulent inflow data in a neutral boundary layer for LESs. The solution domain contained two regions: (1) a driver region, where flow quantities are extracted from a cross-section at a specific downstream location and are recycled into the inlet to generate velocity fluctuations for the model region, and (2) a model region for simulating flow over the target model. Figure 3 shows the computation arrangement.

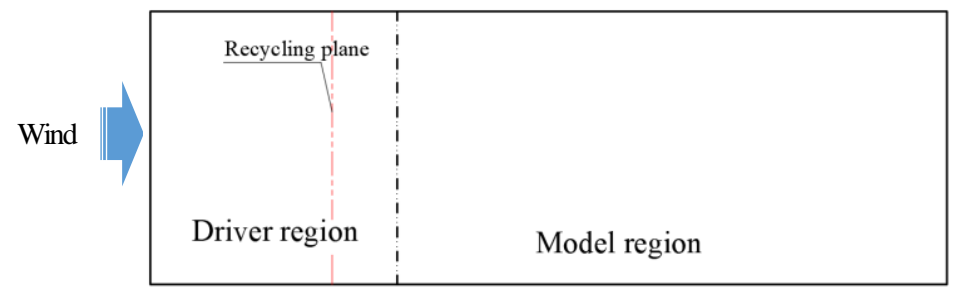

Figure 3. Solution domain setup.

The instantaneous wind velocities of the simulation after recycling to the inflow of the driver region can be calculated as follows [40]:

$$
\begin{gathered}
u_{\text {inlet }}(y, z, t)=U_{\text {inlet }}(z)+\varphi(\theta) \times\{u(y, z, t)-U(y, z)\}_{\text {rec }} \\
v_{\text {inlet }}(y, z, t)=\varphi(\theta) \times\{v(y, z, t)-V(y, z)\}_{\text {rec }} \\
w_{\text {inlet }}(y, z, t)=\varphi(\theta) \times\{w(y, z, t)-w(y, z)\}_{\text {rec }} \\
\varphi=\frac{1}{2}\left\{1-\frac{\tanh \left[\frac{8.0(\theta-1.0)}{0.4(0.3-\theta)+0.7}\right]}{\tanh (8.0)}\right\}
\end{gathered}
$$

where $U_{\text {inlet }}(z)$ is the wind inlet profile from the wind tunnel experiments, which is introduced in Section 3.2.1. $U, V$, and $W$ are mean velocities. $u, v$, and $w$ are instantaneous velocities. The subscripts "inlet" and "rec" denote the variables at the inlet and the recycling planes. $\theta=z / \delta^{\prime} . \varphi$ is the damping factor and $\delta^{\prime}$ is the boundary layer thickness in LES. $\varphi$ ranges from 0 to 1 , as shown in Figure 4. 


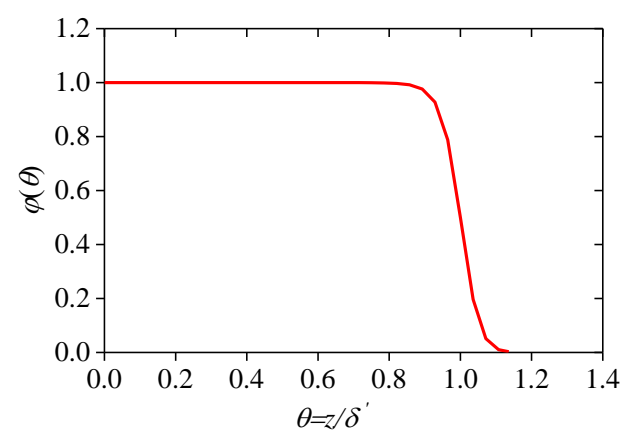

Figure 4. The damping factor $\varphi(\theta)$.

The distance between the recycling plane and the inlet relates to the size of the turbulence structure in the atmospheric boundary layer. The recycling domain should contain the largest scale turbulence structure or at least twice the boundary layer thickness [32]. Therefore, about twice the boundary layer thickness $(2.4 \mathrm{~m})$ was set as the length of the recycling area. Abundant research about LES of flow over building arrays has applied the turbulent inlet over smooth terrains [32,33], while the inlet application over rough terrain is seldom studied. The total drag forces of all buildings and the drag forces of individual buildings are validated with the experiments. This paper arranged the roughness models given by Nozawa and Tamura [41] in the driver region, as shown in Figure 3, and the drag force validation is presented in Section 3.2.4.

\section{Case Configurations and LES Validation}

\subsection{Case Models and Configurations}

Considering the actual urban buildings, as shown in Figure 1, the tested cases were simplified, as shown in Figure 5. The dashed line with an arrow indicates the central axis of the local wind passage. Figure 5a depicts the case of distorted wind passages (DWP), where the central axes of the local wind passage that are not collinear. DWP is selected from one case in the wind tunnel studies of Li et al. [42], and as a validation experiment. Because the cases studied in this experiment were the orthogonal design of the morphological parameters, this case consisted of 23 cuboids, 6 rows, and 4 columns. Figure $5 \mathrm{~b}$ shows the case of the streamlined wind passages (SWP), where the central axes of the local wind passage that are collinear. The same 23 cuboids were adapted in the SWP to ensure that only the wind passage types differed between cases. Three wind passages, between $C_{1}$ and $C_{2}, C_{2}$ and $C_{3}, C_{3}$ and $C_{4}$, named WP1, WP2, WP3 respectively, were designed. The blockage ratio was $1.9 \%$ for both cases.

(a)

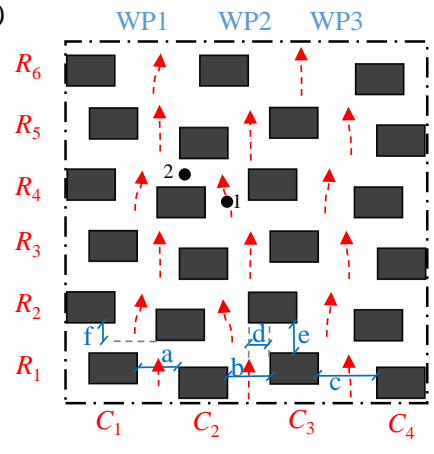

DWP: Distorted wind passages

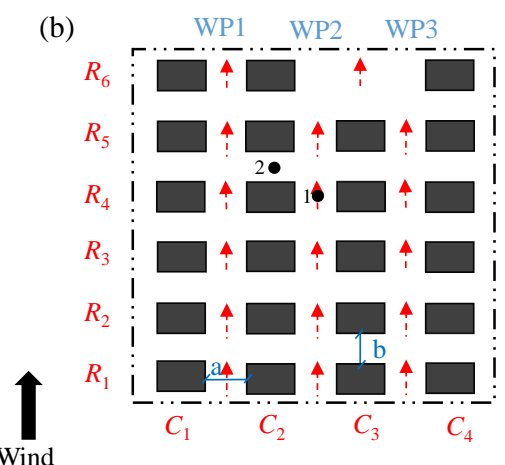

SWP: Streamlined wind passages

Figure 5. Schematic diagrams of model arrangement for cases (a) DWP: distorted wind passages and (b) SWP: streamlined wind passages. The spatial average domain is presented as the area of dot-dashed line with the size of $1.6 \times 1.6 \mathrm{~m}^{2}$. Two points, $\mathrm{P} 1$ and $\mathrm{P} 2$ are selected as representatives to denote the wake and wind passage regions. 
All building models were the same size of $213.7 \mathrm{~mm}$ (length) $\times 144.4 \mathrm{~mm}$ (width) $\times 105 \mathrm{~mm}$ (height). The span-wise distances a, b, c, and d and stream-wise distances e and $f$ for DWP marked in Figure 5 a were $0.186 \mathrm{~m}, 0.186 \mathrm{~m}, 0.256 \mathrm{~m}, 0.100 \mathrm{~m}, 0.126 \mathrm{~m}$ and $0.066 \mathrm{~m}$, respectively. For SWP, the span-wise and stream-wise distances a and b marked in Figure $5 \mathrm{~b}$ were $0.122 \mathrm{~m}$ and $0.186 \mathrm{~m}$. The planar area density $\lambda_{p}$ was $27.5 \%$, and the frontal area density $\lambda_{f}$ is $25 \%$ for both cases. The Symbol $B_{\mathrm{ij}}$ was used to denote the specific individual building model at the $i$ th row and $j$ th column, where the capital letter " $B$ " was the abbreviation of building, $i$ and $j$ represented the row and column number coordinates. The symbols $R_{\mathrm{i}}$, and $C_{\mathrm{j}}$ indicate the buildings at the $i$ th row and the buildings at the $j$ th row. For instance, the second column of buildings, $C_{2}$, comprised six building models: $B_{1,2}, B_{2,2}$, $B_{3,2}, B_{4,2}, B_{5,2}$, and $B_{6,2}$.

The spatial average domain is presented as the area of the dot-dashed line with the size of $1.6 \times 1.6 \mathrm{~m}^{2}(15.2 \mathrm{H} \times 15.2 \mathrm{H})$ in Figure 5. Moreover, to compare the velocities of wake and wind passage regions for both cases, we selected point 1 behind building model $B_{4.2}$ to represent the wake region, denoted as $\mathrm{P} 1$, and point 2 between the building models $B_{4.3}$ and $B_{4.3}$ to represent the wind passage region, denoted as P2.

\subsection{LES Validation}

\subsubsection{Reference Wind Tunnel Experiments}

The reference wind tunnel experiments for DWP were conducted in the small section of the Wind Tunnel and Wave Trough Laboratory of Harbin Institute of Technology. The cross-section of the wind tunnel is $3 \mathrm{~m}$ high and $4 \mathrm{~m}$ wide. The experimental diagram is shown in Figure 6. According to the Load Code for the Design of Building Structures of China (GB 50009-2012), the inflow terrain was of the standard C-type, indicating it was similar to the flow over dense areas such as forested and gentle hilly lands, as well as low-rise and high-rise buildings. The terrain roughness index (in power-law profile) was 0.22 . The fetch length was $19.2 \mathrm{~m}$, and the thickness of the boundary layer for the wind tunnel experiment $\delta$ was about $1.3 \mathrm{~m} \mathrm{(450} \mathrm{m}$ in full scale). The experimental mean wind velocity at the boundary layer height $\delta, U_{\mathrm{g}}$, was $7.98 \mathrm{~m} / \mathrm{s}$. The mean inlet profile $U_{\text {inlet }}(z)$ for LES was measured under the condition of no immersing models.

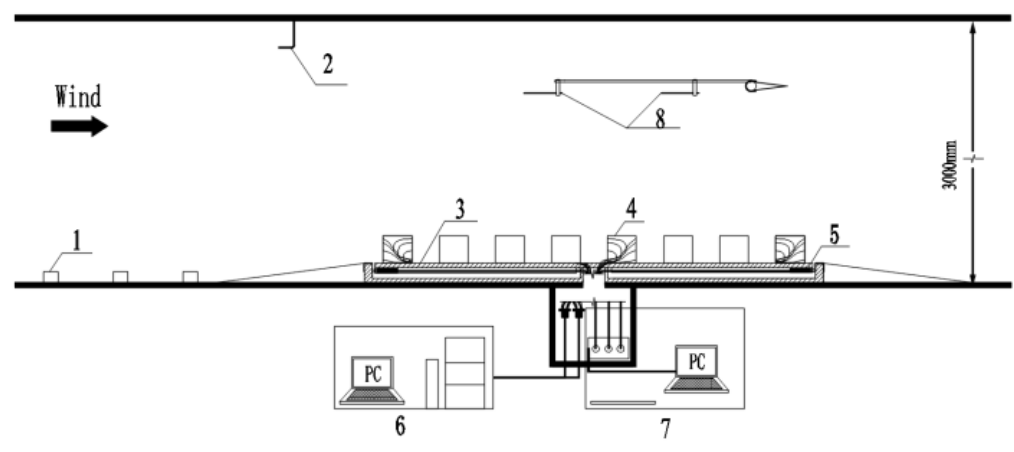

(a)

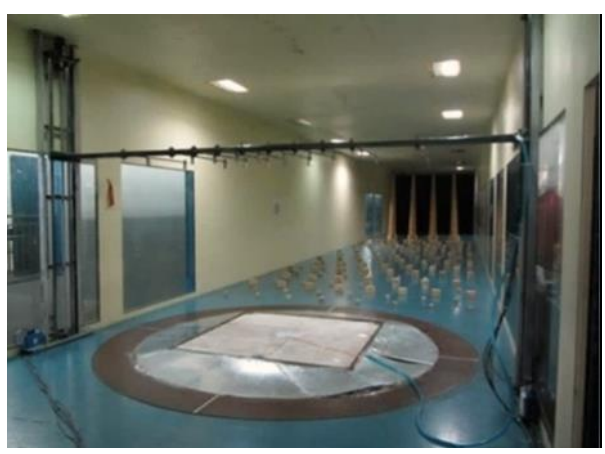

(b)

Figure 6. (a) Schematic diagram of wind tunnel platform. 1. Terrain roughness elements, 2. Pitot-static tube, 3 . Floating device, 4. Pressure module, 5. Force sensor, 6. Pressure scanning valve system, 7. Force sensor system, 8. Hot-wire anemometers. (b) Actual setup in the wind tunnel.

The windward and leeward surface pressures of six individual buildings, represented by the white models in Figure 7a, as well as the reference velocities at a height of $2.7 \mathrm{~m}$, were collected by a pressure scanning system (Miniature Analog Pressure Scanners, SCANIVALV, DSM3400) with an accuracy of $0.08 \%$ and frequency of $312.5 \mathrm{~Hz}$. A floating baseboard, with a planar area of $1.6 \times 1.6 \mathrm{~m}^{2}$, combined with force sensors (Interface, SPI-3LBF) on its span-wise and downwind directions with an accuracy of $0.02 \%$, were used to measure the total drag force of the building array. Force data were collected by acquisition equipment (ME, DSCH) with an accuracy of $0.002 \%$ and frequency of $100 \mathrm{~Hz}$. Two hot-wire probes 
(DENTEC, 55P11) situated $0.2 \mathrm{~m}$ apart were installed on the supporting device at the center of the test section region, as marked by the two cross symbols in Figure $7 \mathrm{a}$, to measure the incoming flow at 10 elevations: $1.5 \mathrm{H}, 2.5 \mathrm{H}, 3.5 \mathrm{H}, 4.5 \mathrm{H}, 5.5 \mathrm{H}, 6.5 \mathrm{H}, 8.5 \mathrm{H}, 10.5 \mathrm{H}, 12.5 \mathrm{H}$ and $14.5 \mathrm{H}$ ( $H$ refers to the model height). The sampling frequency was $1000 \mathrm{~Hz}$, and the accuracy was $0.1 \%$ after calibration. Pressure taps were installed on the windward and leeward surfaces of $C_{2}$ along the wind direction, as shown in Figure $7 \mathrm{~b}$. More details of the experiments can be found in the research of $\mathrm{Li}$ et al. [42].

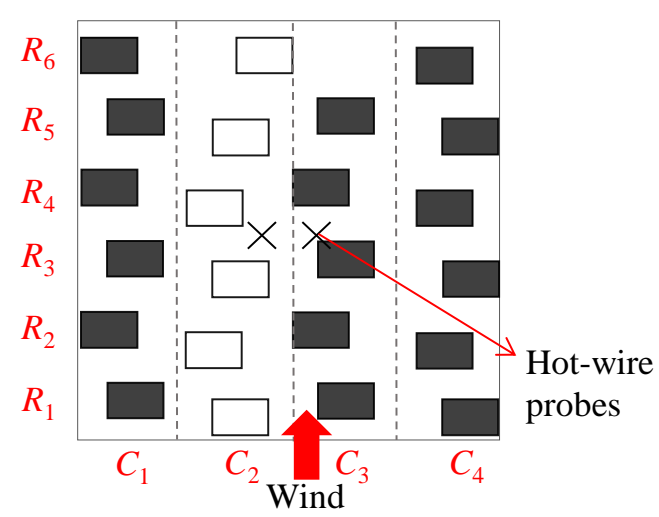

(a)

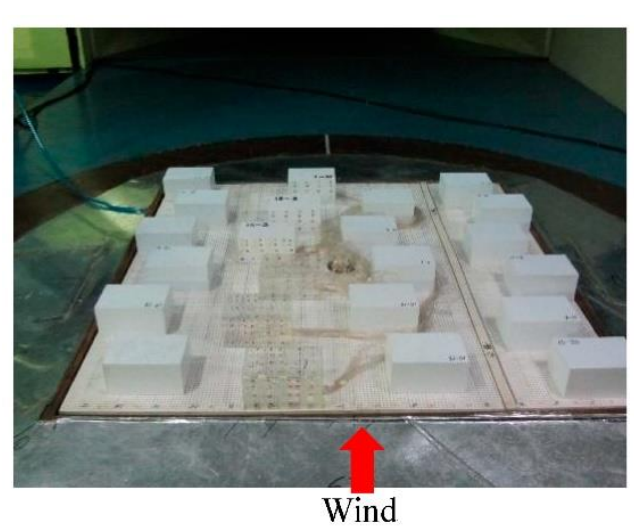

(b)

Figure 7. Schematic diagrams of model arrangement. (a) Plan view. Surface pressure data on the windward and leeward sides of $C_{2}$ were measured. (b) Isometric view. Cross marks denote the horizontal locations of two hot-wire probes.

\subsubsection{Computational Domain and Mesh for LESs}

The dimension of the computational domain was $9.6 \mathrm{~m}(91.4 H)$ long, $3.84 \mathrm{~m}(36.6 H)$ wide, and $1.59 \mathrm{~m}(15.2 H)$ high. Based on building height $H$ and mean velocity at the height of boundary layer $U_{g}$, the Reynolds number was $5.6 \times 10^{4}$. The upwind fetch from the last row of the building models in the driver region to the first obstacle row of the target building array was $24 \mathrm{H}$. The distance from the last row of target buildings to the outlet boundary was $29 \mathrm{H}$. The lateral boundaries were located $9 \mathrm{H}$ from the outer buildings. The above domain sizes fulfill the major simulation requirements as recommended by Tominaga et al. [43]. The size of the roughness elements in the driver region is $120 \mathrm{~mm}$ (length) $\times 120 \mathrm{~mm}$ (width) $\times 100 \mathrm{~mm}$ (height), arranged in a staggered pattern, and the planar area density was $16 \%$.

A nesting mesh was used in the solution domain with the structured mesh, as shown in Figure 8 . For the maximal grid resolution $(H / \Delta, \Delta$ is the minimal grid size), Coceal et al. [18] used uniform grid size $H / \Delta$ of 32 for direct numerical simulation (DNS) of flow over periodic staggered and aligned buildings. Xie et al. [44] suggested that $H / \Delta$ of $15-20$ are sufficient to obtain the total drag force of an actual urban area by adopting 16 for $H_{m} / \Delta . H_{m}$ refers to the mean building height for LES of periodic building arrays of random building heights. Yang et al. [45] investigated the drag forces for flow over buildings with aligned and staggered layouts at very low planar indexes using LESs and set a uniform grid $H / \Delta$ of 6 in wall-modeled LESs and about 50 for wall-resolved LESs. Liu et al. [46] set $H / \Delta$ as 40, 200, 400, and 800 for nine building models to investigate pedestrian level wind speed. 


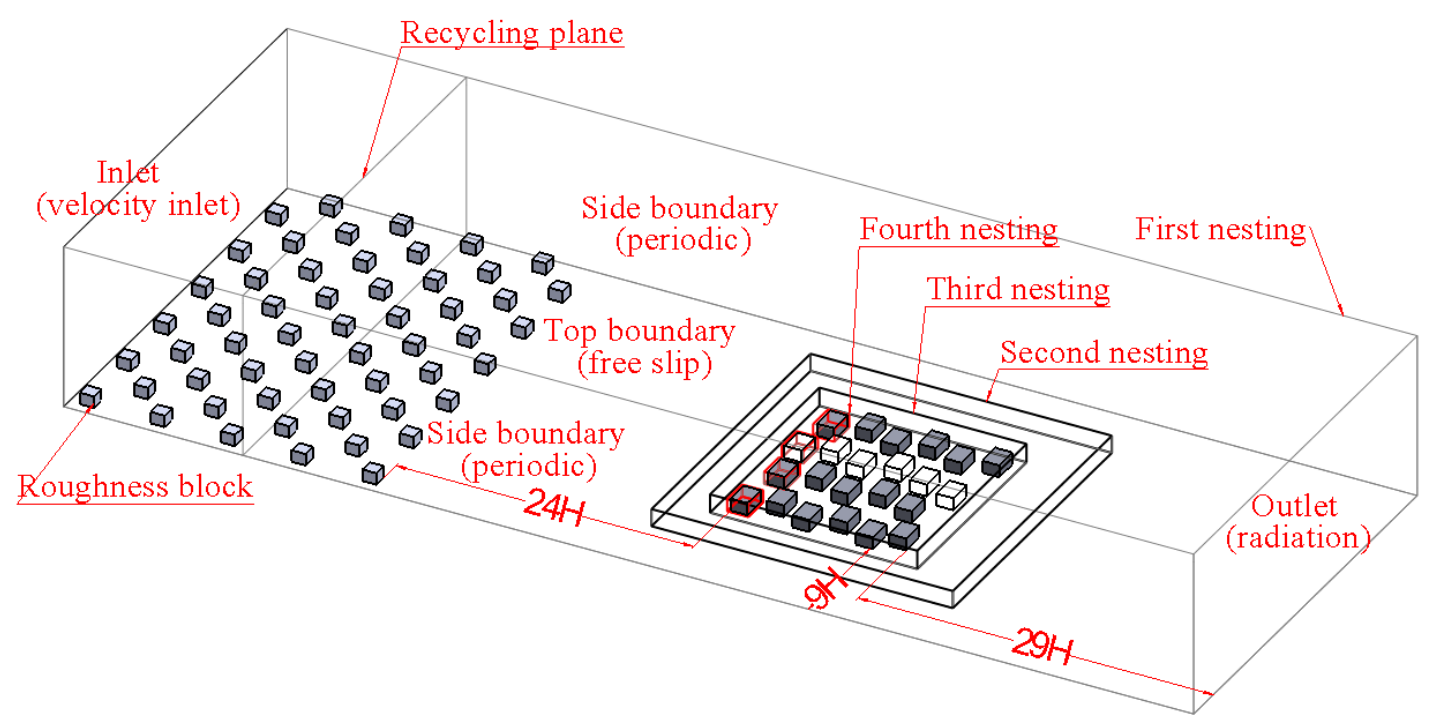

Figure 8. Nesting grid configuration.

This study adopted four layers of nesting meshes within the solution domain to refine the mesh cells around the buildings to a high-resolution level and reduce the total grid number. Uniform grid sizes were used within each nesting mesh. Synthetically considering the limited computing resources and many building models, we set 168 grid points in the vicinity of the first row of buildings that were strongly sheltered by other buildings and intensely exposed to the flow. We used 42 grid points for other buildings (coarse mesh). $\Delta x, \Delta y$, and $\Delta z$ were the grid sizes in $x, y$, and $z$ directions. To check grid dependence, results based on three meshes, which are named as coarse, medium, and fine meshes, are compared. Table 1 and Figure 8 show the configurations of the nesting domains in detail; for example, the first vertical grid near the wall is $0.024 \mathrm{H}(0.5 \mathrm{~m}$ in full scale) for the third nesting domain in the medium mesh. Two-way nesting mode was used in all simulations, i.e., the sub-domain obtained boundary conditions from its upper-class domain through tri-linear interpolation, and the sub-domain influenced its upper-class domain through extrapolation. All simulated results were derived from the nesting with the most refined grid mesh, considering that each nesting domain had a solution. The roughness length $\mathrm{z}_{0}$ in the wall function of Equation (9) for the first nesting domain that contained the roughness elements was set as $0.1 \mathrm{~m}$ in full scale, replicating the setup of Yoshida et al. [24]. For other nesting domains that contained building models, the roughness length $z_{0}$ was set at $0.01 \mathrm{~m}$ in full scale [47]. The simulations were performed on 36 core Intel Xeon Gold $2.30 \mathrm{GHz}$ CPUs with 256 GB RAM. The CPU time for the computations without building array required one day, and the coarse, medium, and fine meshes, required 5, 9 and 13 days, respectively.

Table 1. Detailed configurations of nesting domains.

\begin{tabular}{|c|c|c|c|c|c|c|c|c|}
\hline & Iesh & $H / \Delta x$ & $H / \Delta y$ & $H / \Delta z$ & $\begin{array}{c}\text { The First } \\
\text { Vertical Grid }\end{array}$ & $\begin{array}{c}\text { Mesh Domain } \\
\text { (Length } \times \text { Width } \times \text { Height) }\end{array}$ & Quantity & $\begin{array}{l}\text { Grid Cells } \\
\text { (Millions) }\end{array}$ \\
\hline \multirow{3}{*}{$\begin{array}{l}\text { Coarse } \\
\text { mesh }\end{array}$} & First nesting & 5.25 & 5.25 & 10.5 & $0.095 H$ & $91.4 \mathrm{H} \times 36.6 \mathrm{H} \times 13 \mathrm{H}$ & 1 & \multirow{3}{*}{19.6} \\
\hline & Second nesting & 10.5 & 10.5 & 21 & $0.048 \mathrm{H}$ & $24.4 H \times 24.4 H \times 3 H$ & 1 & \\
\hline & Third nesting & 21 & 21 & 42 & $0.024 H$ & $16.8 H \times 16.8 H \times 1.5 H$ & 1 & \\
\hline \multirow{4}{*}{$\begin{array}{l}\text { Medium } \\
\text { mesh }\end{array}$} & First nesting & 5.25 & 5.25 & 10.5 & $0.095 H$ & $91.4 \mathrm{H} \times 36.6 \mathrm{H} \times 13 \mathrm{H}$ & 1 & \multirow{4}{*}{24.9} \\
\hline & Second nesting & 10.5 & 10.5 & 21 & $0.048 \mathrm{H}$ & $24.4 H \times 24.4 H \times 3 H$ & 1 & \\
\hline & Third nesting & 21 & 21 & 42 & $0.024 H$ & $16.8 H \times 16.8 H \times 1.5 H$ & 1 & \\
\hline & Fourth nesting & 84 & 84 & 168 & $0.006 \mathrm{H}$ & $1.7 H \times 2.3 H \times 1 H$ & 4 & \\
\hline \multirow{4}{*}{ Fine mesh } & First nesting & 5.8 & 5.8 & 11.7 & $0.085 H$ & $91.4 H \times 36.6 H \times 13 H$ & 1 & \multirow{4}{*}{32.6} \\
\hline & Second nesting & 11.7 & 11.7 & 23.3 & $0.043 \mathrm{H}$ & $24.4 H \times 24.4 H \times 3 H$ & 1 & \\
\hline & Third nesting & 23.3 & 23.3 & 46.7 & $0.021 H$ & $16.8 \mathrm{H} \times 16.8 \mathrm{H} \times 1.5 \mathrm{H}$ & 1 & \\
\hline & Fourth nesting & 93.3 & 93.3 & 186.7 & $0.005 H$ & $1.7 H \times 2.3 H \times 1 H$ & 4 & \\
\hline
\end{tabular}




\subsubsection{Evaluation Metrics}

This study used five validation metrics to assess the prediction accuracy of LESs with the wind tunnel data (Table 2): hit rate (q), a factor of 2 of the observation (FAC2), fractional bias (FB), normalized mean square error (NMSE), and Pearson correlation coefficient (R). The metrics are defined as follows:

$$
\begin{gathered}
\mathrm{q}=\frac{1}{N} \sum_{i=1}^{N} n_{i}, n_{i}=\left\{\begin{array}{ll}
1, & \frac{P_{i}-M_{i}}{M_{i}} \mid \leq D_{q} \\
0, & \text { else }
\end{array} \mid\right. \\
\mathrm{FAC} 2=\frac{1}{N} \sum_{i=1}^{N} n_{i}, n_{i}= \begin{cases}1, & 0.5 \leq \frac{P_{i}}{M_{i}} \leq 2 \\
0, & \text { else }\end{cases} \\
\mathrm{FB}=\frac{\left[M_{i}\right]-\left[P_{i}\right]}{0.5\left(\left[M_{i}\right]+\left[P_{i}\right]\right)} \\
\mathrm{R}=\frac{\left[\left(M_{i}-\left[M_{i}\right]\right)\left(P_{i}-\left[P_{i}\right]\right)\right]}{\sqrt{\left(M_{i}-\left[M_{i}\right]\right)^{2}} \times \sqrt{\left(P_{i}-\left[P_{i}\right]\right)^{2}}}
\end{gathered}
$$

where $M_{\mathrm{i}}$ and $P_{\mathrm{i}}$ are the measured data from the experiments and the predicted data from LESs for sample $i$ and $N$ is the number of samples. The square bracket indicates the

\begin{tabular}{|c|c|c|c|c|c|c|}
\hline \multicolumn{2}{|c|}{ Metrics } & $\mathbf{q}$ & FAC2 & FB & NMSE & $\mathbf{R}$ \\
\hline \multicolumn{2}{|c|}{ Ideal value } & 1 & 1 & 0 & 0 & 1 \\
\hline \multicolumn{2}{|c|}{ Acceptance criteria } & $>0.66$ & $>0.5$ & $<0.15$ & $<4$ & $>0.8$ \\
\hline \multirow[t]{2}{*}{$U$} & $\begin{array}{l}\text { With } \\
\text { models } \\
\text { immersed }\end{array}$ & 1 & 1 & 0 & 0.01 & 0.89 \\
\hline & $\begin{array}{l}\text { Without } \\
\text { models } \\
\text { immersed } \\
\text { With }\end{array}$ & 1 & 1 & 0.02 & 0 & 0.89 \\
\hline \multirow[t]{2}{*}{$I_{\mathrm{u}}$} & $\begin{array}{l}\text { models } \\
\text { immersed }\end{array}$ & 0.8 & 1 & 0.08 & 0.02 & 0.86 \\
\hline & $\begin{array}{l}\text { Without } \\
\text { models } \\
\text { immersed }\end{array}$ & 0.9 & 1 & 0.02 & 0.03 & 0.86 \\
\hline
\end{tabular}
average of all samples. The ideal values are 1 for FAC2 and q, and 0 for FB and NMSE. Previous research suggests that $\mathrm{q}>0.8, \mathrm{FAC} 2>0.5,|\mathrm{FB}|<0.3, \mathrm{NMSE}<4$, and $\mathrm{R}>0.8$ are accepted [48-50]. The threshold value for $D_{\mathrm{q}}$ is set to 0.25 .

Table 2. Validation metrics for wind speeds $U$ and turbulence intensities $I_{\mathrm{u}}$ for LESs.

\subsubsection{Validation and Grid Sensitivity Analysis}

Validation of Velocity

The turbulent characteristics are examined without and with the immersed buildings. Flow characteristics obtained from LESs and the experiments without and with immersing building array are shown in Figures 9 and 10, respectively. As shown in Figure 9a, the experimental results are in good agreement with the power-law profile, indicating that the inflow terrain accurately reproduced the standard C-type terrain profile at the test section. 


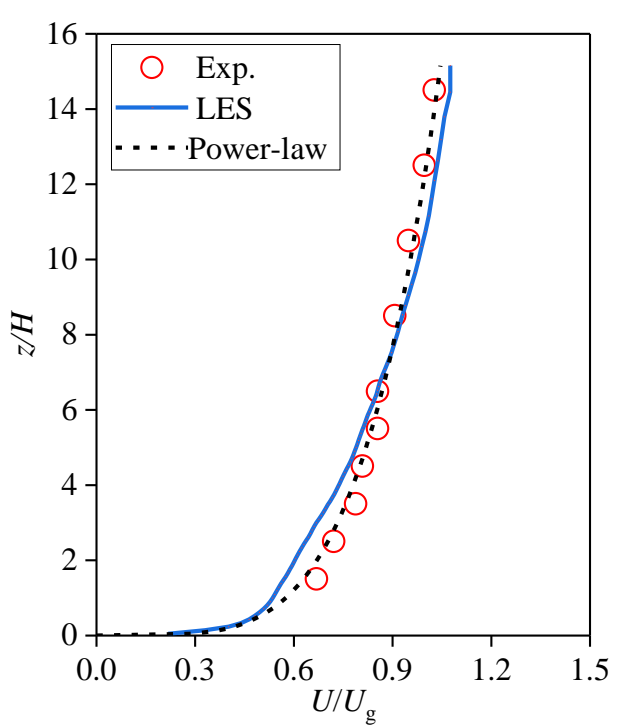

(a)

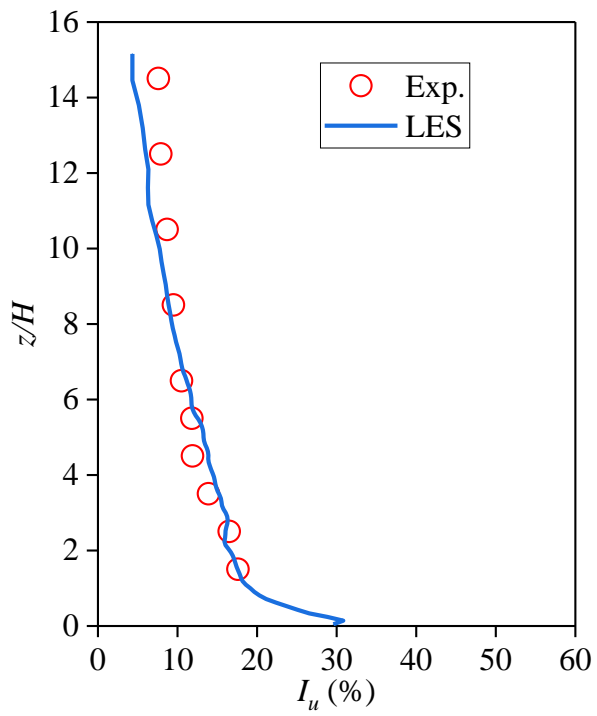

(b)

Figure 9. Approaching flow characteristics obtained from LES and experiment without immersing building models. (a) Normalized stream-wise mean velocity $U / U_{\mathrm{g}}$, where $U_{\mathrm{g}}$ is mean wind velocity at boundary layer height $\delta$ for wind tunnel experiment. (b) Stream-wise turbulence intensity $I_{\mathrm{u}}$.

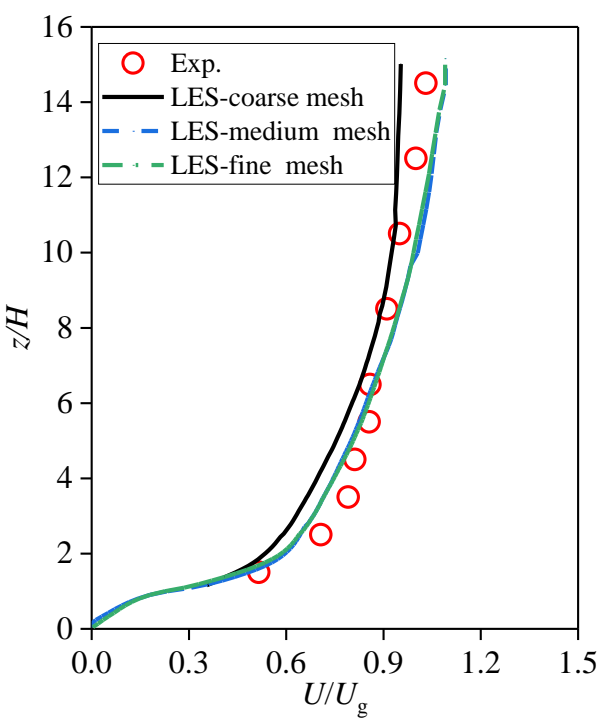

(a)

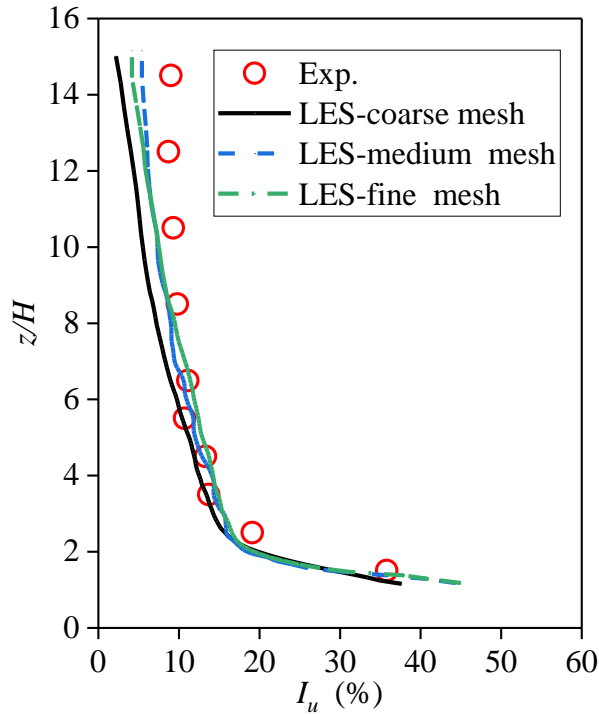

(b)

Figure 10. Approaching flow characteristics obtained from LES and experiment with models mounted. (a) $U / U_{\mathrm{g}}$ (b) $I_{\mathrm{u}}$.

Figure 10 shows the calculated results based on three grid systems. The differences in wind velocity and turbulence intensity between the medium and fine meshes are more significant than between coarse and medium meshes. Table 2 lists the evaluation metrics based on the medium mesh. It is shown that all the evaluation metrics satisfy the criteria, indicating that LES-medium mesh is sufficient to obtain mean and instantaneous flow fields. For the numerical model, the boundary layer height $\delta^{\prime}$ equals $1.4 \mathrm{~m}(480 \mathrm{~m}$ in full scale), and the wind velocity at the boundary layer height, denoted as $U^{\prime}{ }^{\prime}$, is $8.30 \mathrm{~m} / \mathrm{s}$.

Stream-wise power spectral densities at the height of $1.5 \mathrm{H}$ and $10.5 \mathrm{H}$ without immersing building models obtained from LES and experimental results were computed 
and compared with the von Karman spectrum, as shown in Figure 11. The von Karman spectrum is:

$$
\frac{\mathrm{fS}_{\mathrm{u}}}{\sigma_{\mathrm{u}}^{2}}=\frac{4\left(\mathrm{fL}_{\mathrm{u}} / \mathrm{U}\right)}{\left(1+70.8\left(\mathrm{fL}_{\mathrm{u}} / \mathrm{U}\right)^{2}\right)^{5 / 6}}
$$

where $f$ is the frequency and $L_{u}$ is the stream-wise integral length, calculated by Taylor's hypothesis [51]. $S_{\mathrm{u}}$ is the power spectral density of the instantaneous stream-wise velocity, and $\sigma_{\mathfrak{u}}$ is the standard deviation of the stream-wise velocity. LES results coincide well with the experiments and the von Karman spectrum when the normalized frequency $f L_{\mathrm{u}} / U$ was less than unity. This signifies that mean and turbulent characteristics of the atmospheric boundary layer in the wind tunnel are well reproduced and resolved by LES. The LES spectra decrease significantly when the frequency $f L_{u} / U$ is more than unity, which means that the SGS model solves the motions of frequency higher than unity.

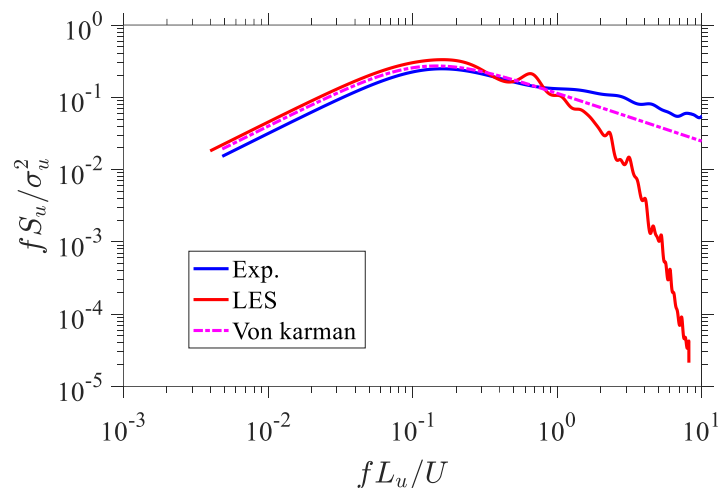

(a)

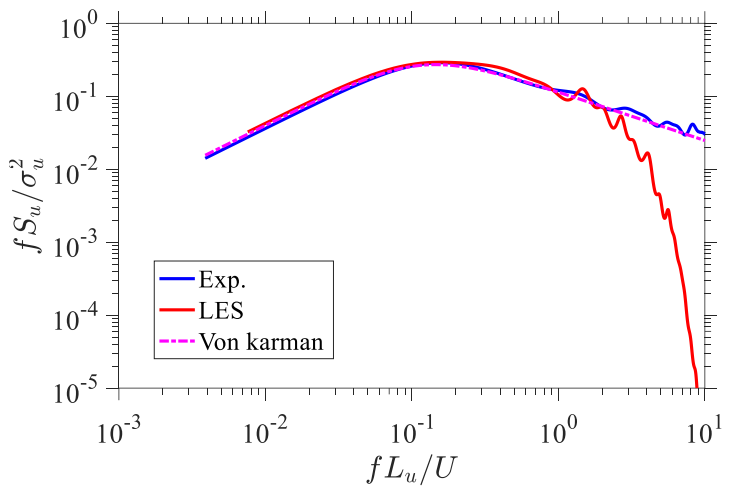

(b)

Figure 11. Stream-wise power spectral densities at (a) $z=1.5 \mathrm{H}$ and (b) $z=10.5 \mathrm{H}$ with immersing building models.

Validation of Drag Force

The form drag force of an individual building model $D_{0}$ can be calculated as:

$$
D_{0}=\iint\left(p_{f}-p_{b}\right) d A_{f 0}
$$

where $p_{\mathrm{f}}$ and $p_{\mathrm{b}}$ are the pressures of the leeward and windward surfaces of the building model. $A_{\mathrm{f} 0}$ is the frontal area of the individual building. The drag coefficient of an individual building, $C_{d 0}$, is computed as [52]:

$$
C_{d 0}=\frac{2 D_{0}}{\rho U_{g}^{2} A_{f 0}}
$$

where $U_{\mathrm{g}}$ indicates mean wind velocity at boundary layer height.

The drag force of the whole building array $C_{d t}$ is calculated as:

$$
C_{d t}=\frac{2 D_{t}}{\rho U_{g}^{2} A_{f t}}
$$

where $D_{\mathrm{t}}$ is the total drag force of the building array, and $A_{\mathrm{ft}}$ is the sum of the frontal area of all individual buildings. $D_{\mathrm{t}}$ can be measured via the force sensors installed on the floating device or the sum of all the individual form drag $D_{0}$ within the building array for blunt body [53].

Figure 12 shows the comparisons of $C_{d 0}$ of building models at the second column and $C_{d t}$ among the wind tunnel experiments, as well as LES results based on three grid systems. For the building model at the first row $B_{1,2}$, the $C_{d 0}$ values for LES-coarse mesh were underestimated, almost by 100\%, while for LES-medium and LES-coarse meshes, 
their differences compared with experimental results are minor. Therefore, these results indicate that the LES-medium mesh is appropriate for predicting the mean wind velocities, the turbulent intensities, and the drag forces of individual buildings.

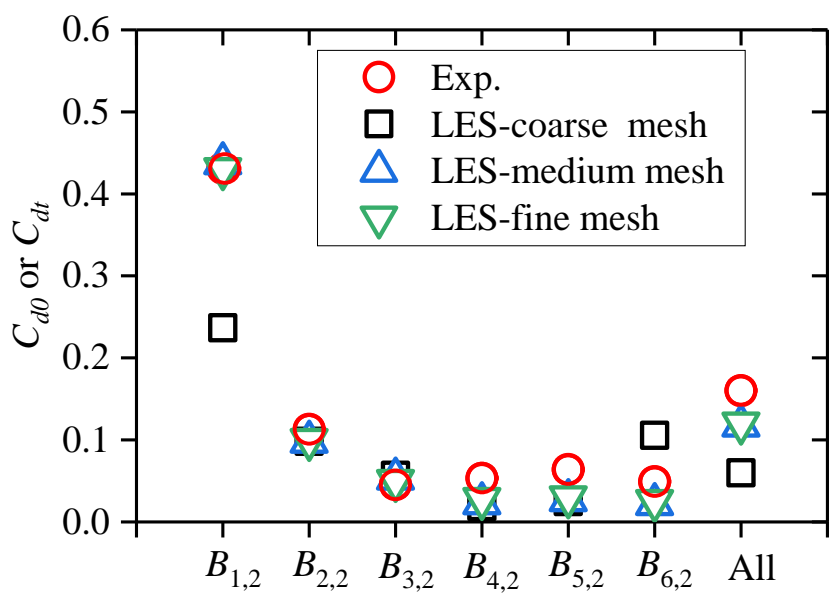

Figure 12. Comparisons of $C_{d 0}$ for six pressure models $C_{2}$ and $C_{d t}$ in DWP among wind tunnel experiments and LES meshes.

$C_{d 0}$ values for LES-medium mesh are consistent between LES and the wind tunnel experiment results, although LES slightly underestimates the drag coefficients of downstream building models. The average absolute deviation of all six $C_{d 0}$ values is 0.021 . The total drag force by adding all the form drag of an individual building coincides with the drag force measured by the floating element in the experiment, with an average absolute deviation of 0.04. Table 3 shows the validation metrics for the drag characteristics. All of the metrics satisfy the acceptance criteria except for q. The probable reason for this is that wall modeling limits the predictions of small-scale flow separations and reattachments originating from the building surfaces [54].

Table 3. Validation metrics for drag characteristics based on LES-medium mesh.

\begin{tabular}{llllll}
\hline Metric & $\mathbf{q}$ & FAC2 & FB & NMSE & R \\
\hline Ideal value & 1 & 1 & 0 & 0 & 1 \\
Acceptance criteria & $>0.8$ & $>0.5$ & $<0.15$ & $<4$ & $>0.8$ \\
$C_{d 0}$ and $C_{d t}$ & 0.57 & 0.57 & 0.04 & 0.06 & 0.99 \\
\hline
\end{tabular}

\section{Numerical Results and Data Analysis}

\subsection{Flow Characteristics}

\subsubsection{Mean Flow Characteristics}

It is necessary to compare how the flow over DWP differs from the flow over SWP. Figure 13 compares the time-averaged flow fields depicted by streamlines of stream-wise mean velocity $U$ at the heights of $0.06 \mathrm{H}$ and $0.5 \mathrm{H}$ above the ground for both cases. The wind streamlines were drawn at a lower resolution for better clarity. 

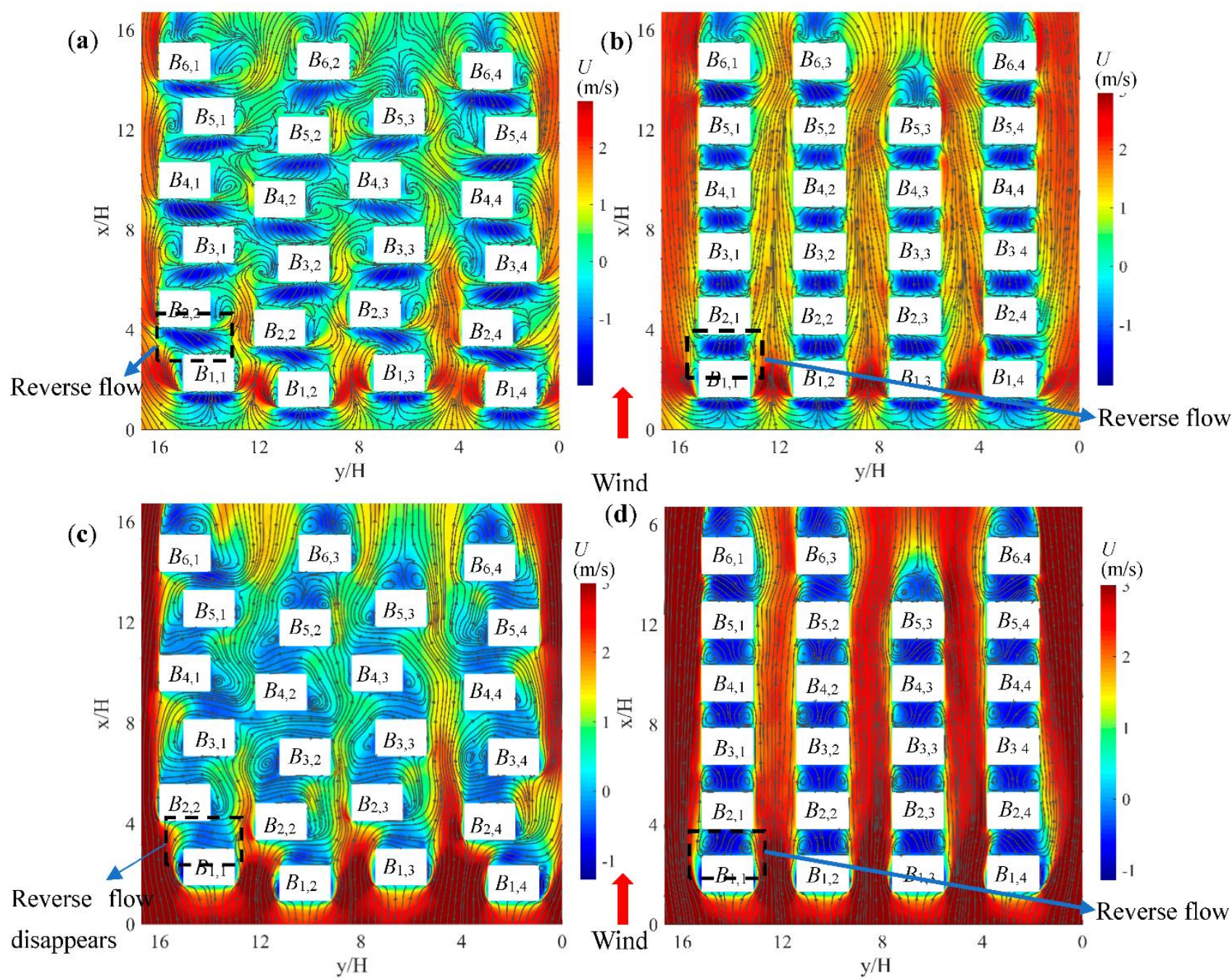

Figure 13. Contours of stream-wise mean velocity $U$ with streamlines at $z=0.06 H$ for (a) DWP (b) SWP and at $z=0.5 H$ for (c) DWP (d) SWP.

For both cases, at the height of $0.06 \mathrm{H}$, there are strong reverse flows at the wake region of the building models. The reverse flows diverge on the leading edge and converge on the leeward surface. A pair of counter-rotating flows behind the last row of buildings is observed similarly to the wake flow pattern over a single building. This flow pattern is also observed behind buildings $B_{5,2}$ and $B_{5,3}$ because the open area behind them causes the recovery of the flow state. For DWP, the side vortices of the buildings obstruct the airflow in the wind passage region, while the side vortices disappear for SWP, resulting in stronger velocities in the wind passage than SWP.

With the increase of height to $0.5 \mathrm{H}$, for DWP, the incoming high-momentum fluid is diverted into the wake region, so the reverse flow mostly disappears, and the span-wise airflow is detected instead, as evidenced by streamlines perpendicular to the wind direction. For SWP, the recirculating vortices still dominate the wake flow.

Figure 14 shows the span-wise mean velocities $V$ at $0.06 \mathrm{H}$ and $0.5 \mathrm{H}$ for both cases. $V$ of DWP in the wake region is about $1 \mathrm{~m} / \mathrm{s}$ higher than SWP, indicating the strong span-wise airflow in the wake region of DWP. The side vortices are present for the DWP, blocking the airflow in the wind passage region, while they are not present for SWP, indicating that SWP reaches higher velocities than DWP. 
(a)

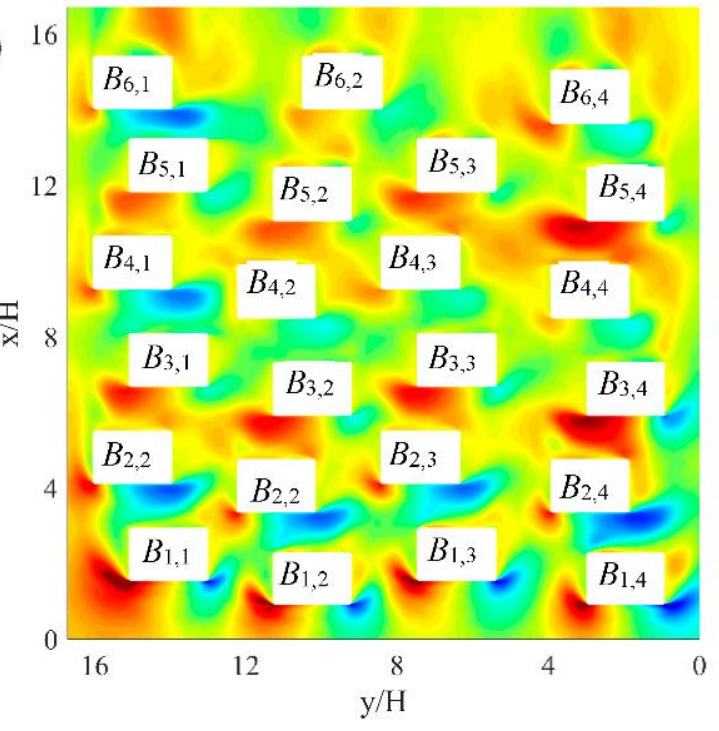

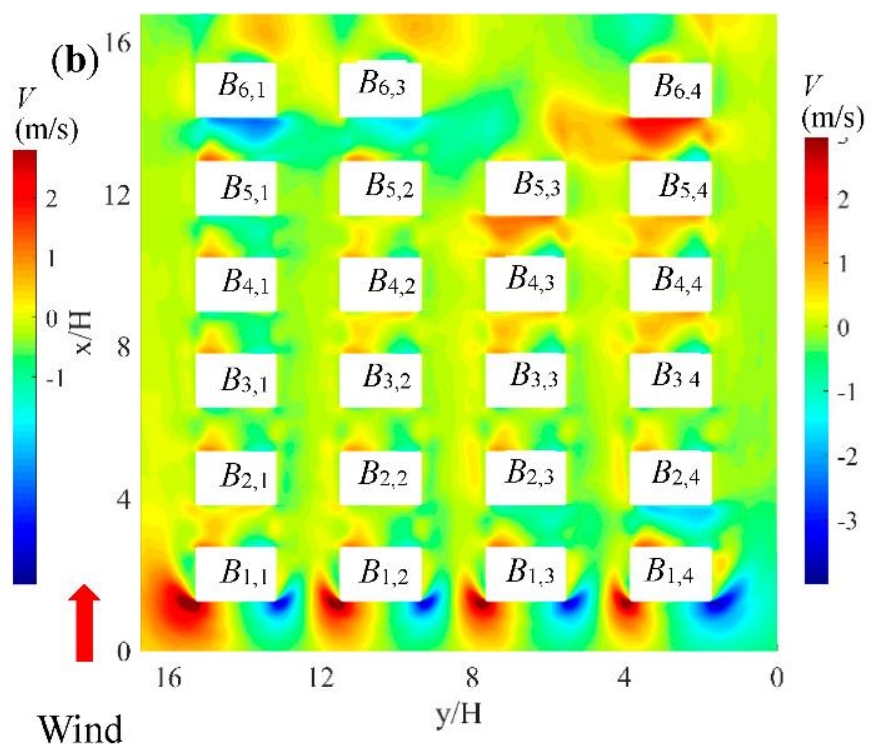

Wind (c)

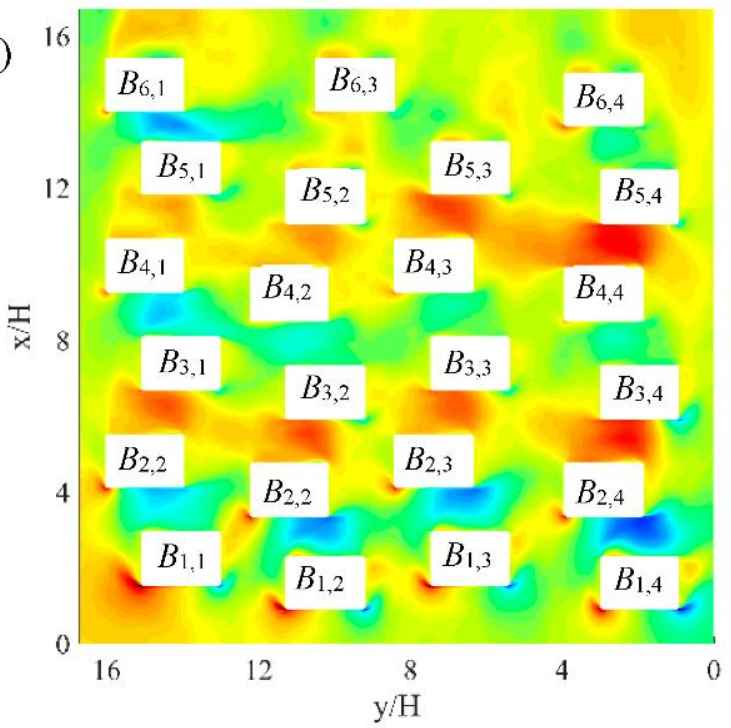

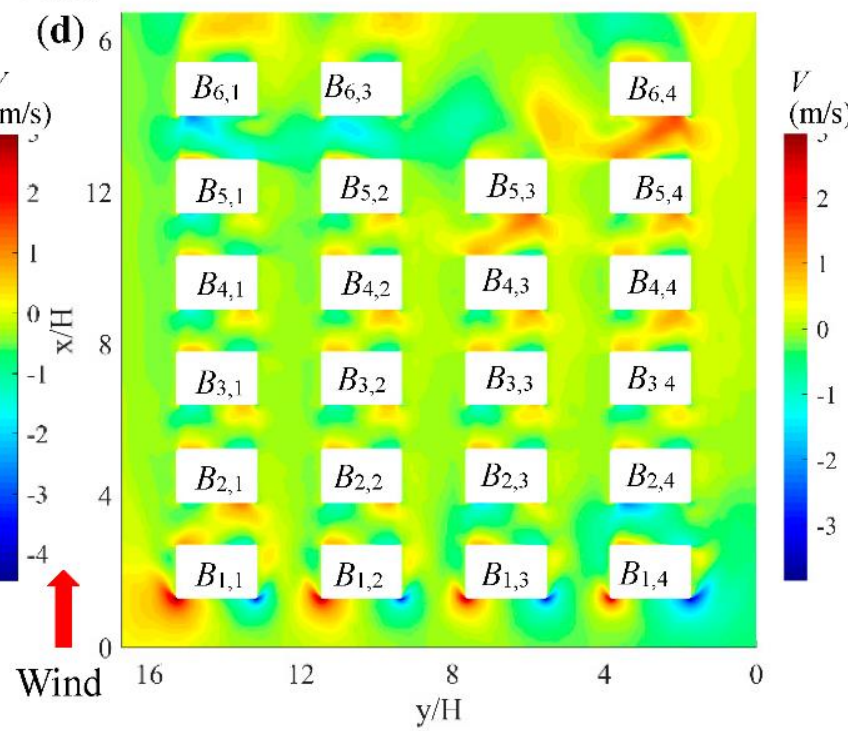

Figure 14. Contours of span-wise mean velocity $V$ at $z=0.06 \mathrm{H}$ for (a) DWP (b) SWP and at $z=0.5 \mathrm{H}$ for (c) DWP (d) SWP.

Figure 15 further displays $U$ and $V$ at $\mathrm{P} 1$ (the wake region behind the building model $B_{4,2}$ centerline) and P2 (wind passage region between building models $B_{4,2}$ and $B_{4,3}$ ) for both cases. The inflow $U$ is attenuated to varying degrees below the height of $3 \mathrm{H}$ at $\mathrm{P} 1$ and P2. Negative mean velocities are observed at $z / H<0.58$ for DWP and $z / H<0.75$ for SWP. The negative velocities of DWP are stronger than SWP at P1 within the urban canopy, while above the urban canopy, the $U$ of SWP exceeds DWP. The $V$ of DWP is 0.02 times of $U_{\mathrm{g}}$, whereas it goes up to 0.15 times for SWP. In the wind passage region (P2), the $U$ for DWP decreased more than for SWP. The $V$ is nearly zero for SWP while it increases to almost 0.08 times the value of $U_{\mathrm{g}}$ at half of the building height for DWP, indicating the existence of span-wise airflow for both the wake and wind passage regions for DWP. Minor differences were observed for the mean vertical velocities $W$ for both cases, so they are not discussed in this study. 


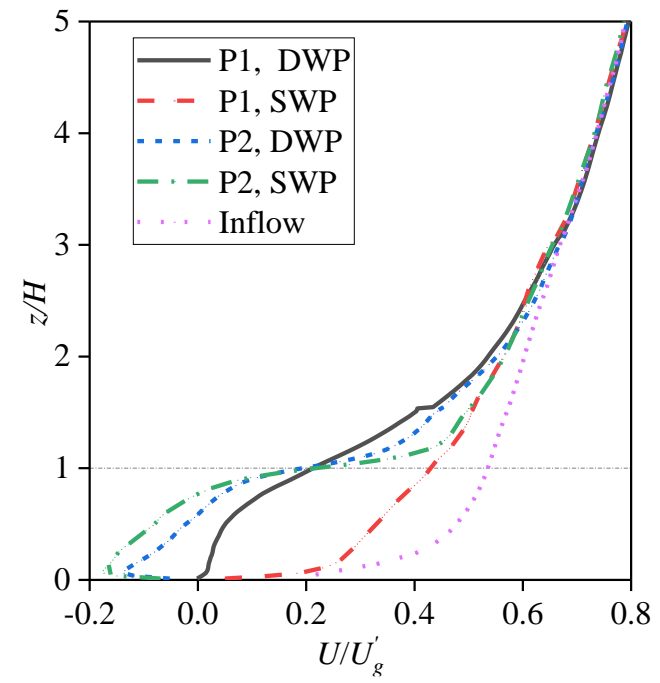

(a)

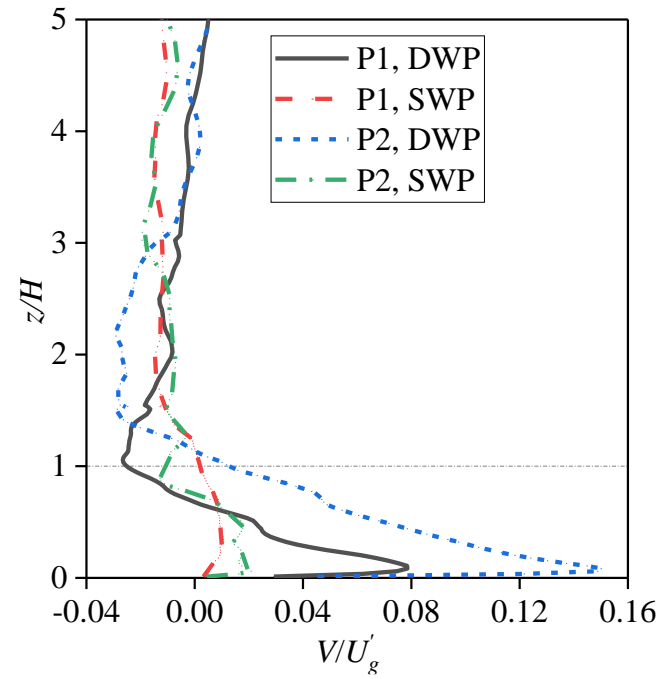

(b)

Figure 15. (a)The $U$ and (b) $V$ profiles at P1 (the wake region behind the building model $B_{4,2}$ centerline) and P2 (wind passage region between building models $B_{4,2}$ and $\left.B_{4,3}\right)$.

\subsubsection{Unsteady Flow Characteristics}

The Reynolds and dispersive stresses are generated by the temporal and spatial average of the $\mathrm{N}$-S equation to represent the turbulence and spatial correlations in the timeaveraged flow. The instantaneous velocity components, $u_{\mathrm{i}}$ can be divided into three components:

$$
u_{i}=\left\langle U_{i}\right\rangle+\widetilde{U}_{i}+u_{i}^{\prime}
$$

where $u_{\mathrm{i}}$ is the instantaneous velocity component. $U_{i}=\overline{u_{i}}$ is the mean velocity, and the overbar indicates the time-averaged operator. $\left\langle U_{i}\right\rangle$ is the time and space averaged velocity, and the angle brackets denote the spatially averaged operator; $\widetilde{U}_{i}=U_{i}-\left\langle U_{i}\right\rangle$ is the spatial variation of the time-mean velocity; $u_{i}^{\prime}=u_{i}-U_{i}$ is the turbulent fluctuation.

Figure 16 compares the profiles of normalized Reynolds stresses, $-\left\langle\overline{u^{\prime} w^{\prime}}\right\rangle,\left\langle\overline{u^{\prime 2}}\right\rangle,\left\langle\overline{v^{\prime 2}}\right\rangle$, and $\left\langle\overline{w^{\prime 2}}\right\rangle$, for both cases. The area applied for horizontal average was located at the center area with a size of $1.6 \times 1.6 \mathrm{~m}^{2}$, as shown in Figure 5 a. Roughly the same trends were found between the two cases. Reynolds stresses as well as TKE peak at the same height of $1.13 H$. The peaks in the $-\left\langle\overline{u^{\prime} w^{\prime}}\right\rangle$ stress for DWP are stronger than that of SWP because of a more intense shear layer over DWP. The range that the buildings affect Reynolds stresses is below the height of $3 H$. Above the canopy, the TKE and all Reynolds stress components of DWP are slightly larger than those of SWP, indicating the rougher surface conditions caused by DWP. Within the urban canopy, due to the strong recirculation vortices in SWP, the changing trends of $\left\langle\overline{u^{\prime 2}}\right\rangle$ and $\left\langle\overline{v^{\prime 2}}\right\rangle$ for both cases below the height of $0.95 \mathrm{H}$ re different.

Figure 17 shows the profiles of dispersive stresses, $-\langle\widetilde{U} \widetilde{W}\rangle,\left\langle\widetilde{U}^{2}\right\rangle,\left\langle\widetilde{V}^{2}\right\rangle$, and $\left\langle\widetilde{W}^{2}\right\rangle$, within and immediately above the urban canopy for the two cases. Above the building height, all dispersive stresses of DWP decrease much more slowly than SWP, indicating significant spatial inhomogeneity of time-averaged velocity for DWP. Within the urban canopy, $-\langle\widetilde{U} \widetilde{W}\rangle$ and $\left\langle\widetilde{U}^{2}\right\rangle$ of SWP are larger than those of DWP, and the $\left\langle\widetilde{U}^{2}\right\rangle$ of SWP is up to 2.5 times of that of DWP at the height of $0.55 \mathrm{H}$, because the streamlined wind passages cause strong spatial inhomogeneity in the mean velocity $U$, which can be seen in Figure 13 . The $\left\langle\widetilde{V}^{2}\right\rangle$ of DWP is bigger than that of SWP, due to more significant $V$ in the wake of buildings of DWP, which can be seen in Figure 14. 


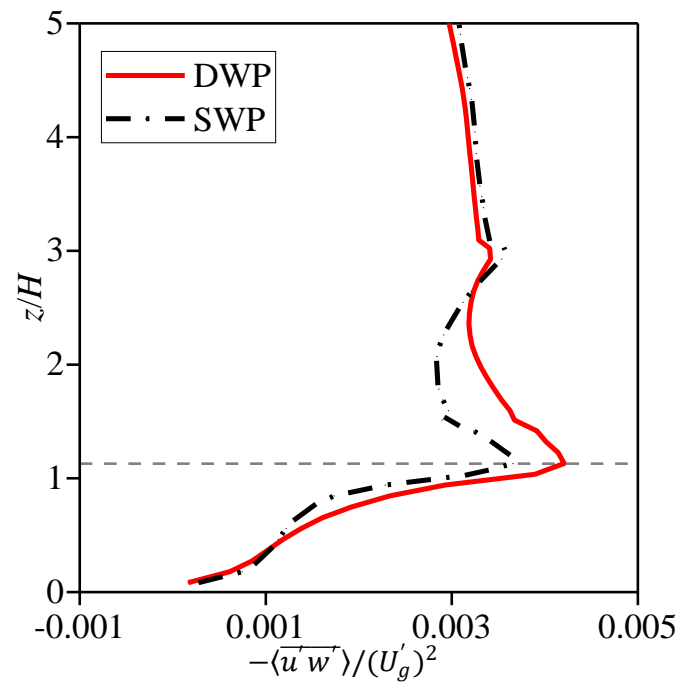

(a)

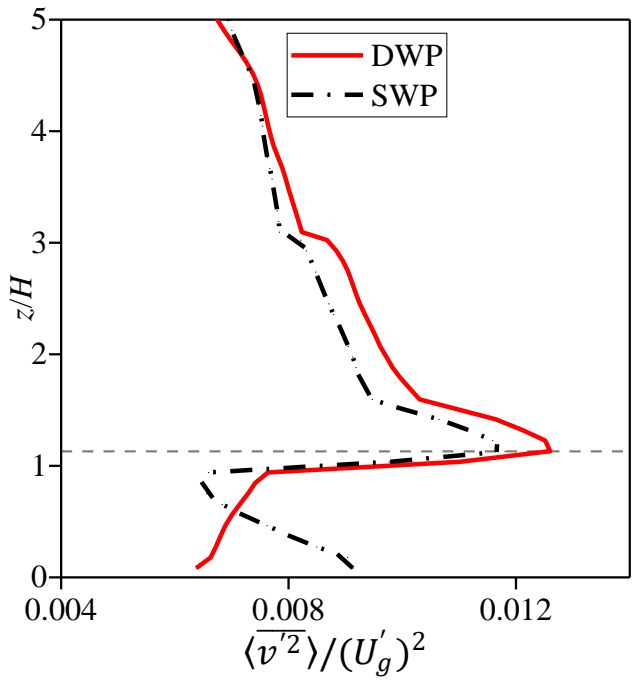

(c)

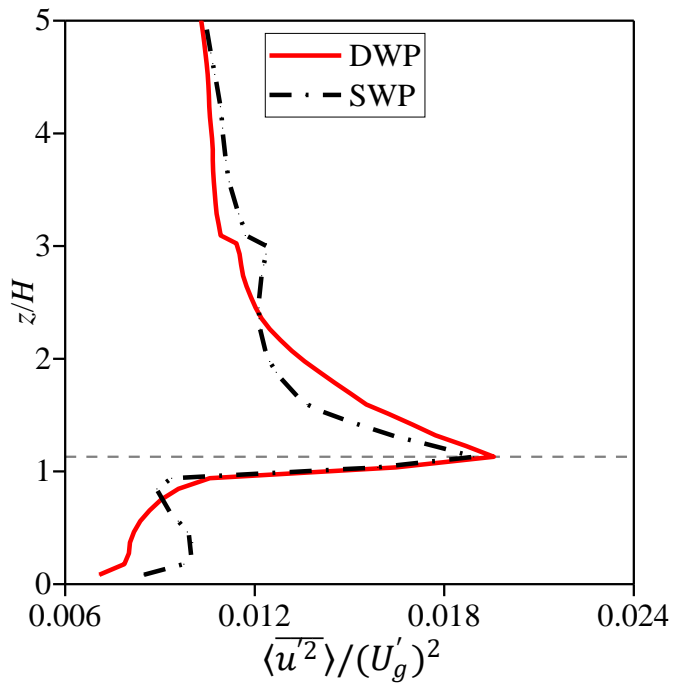

(b)

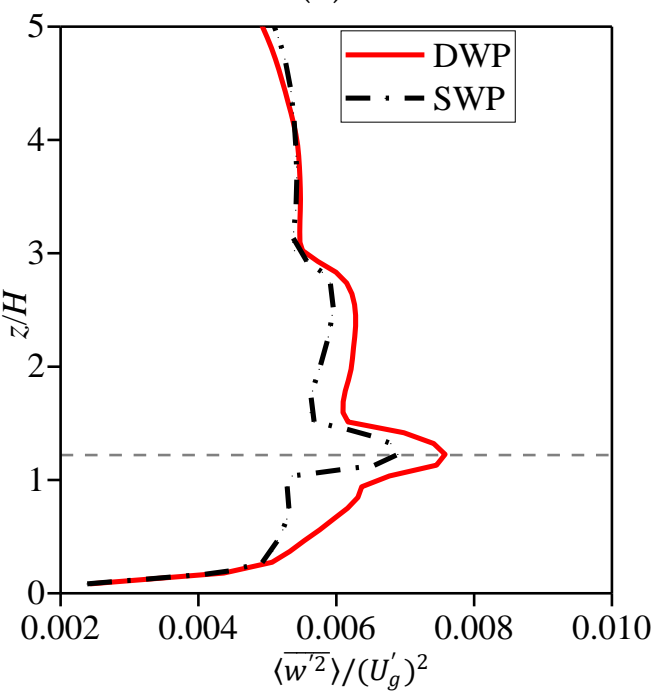

(d)

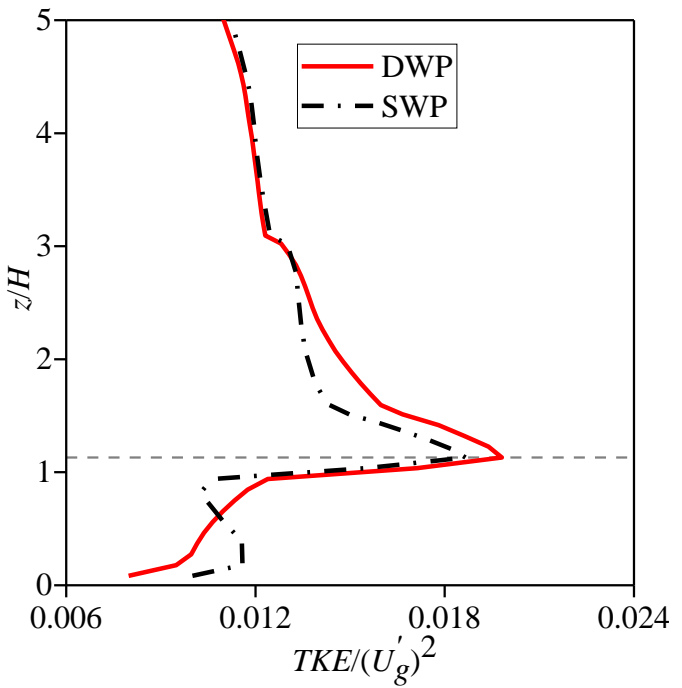

(e)

Figure 16. Reynolds stress components (a) $-\left\langle\overline{u^{\prime} w^{\prime}}\right\rangle,(\mathbf{b})\left\langle\overline{u^{\prime 2}}\right\rangle,(\mathbf{c})\left\langle\overline{v^{\prime 2}}\right\rangle,(\mathbf{d})\left\langle\overline{w^{\prime 2}}\right\rangle$ and (e) $T K E$, normalized by $\left(U_{g}^{\prime}\right)^{2}$. 


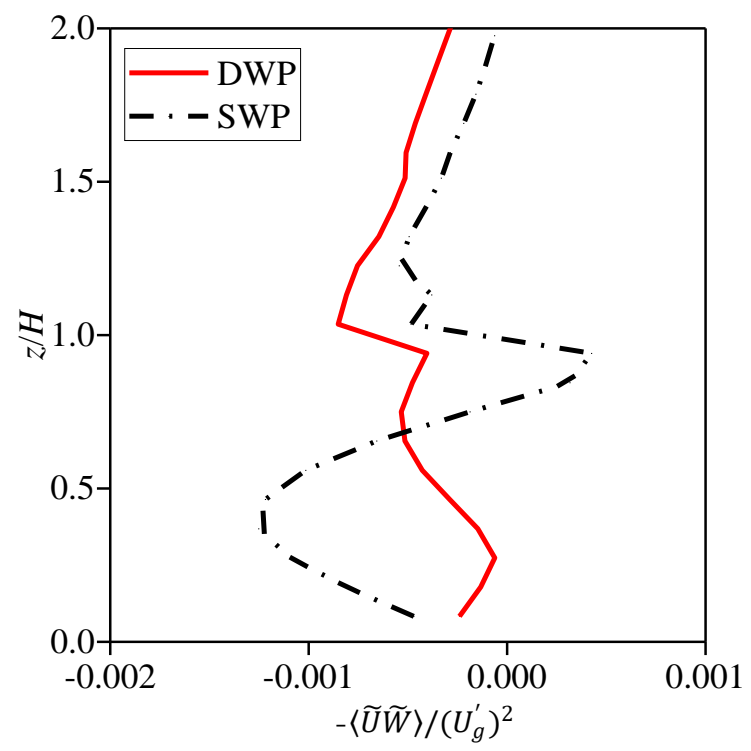

(a)

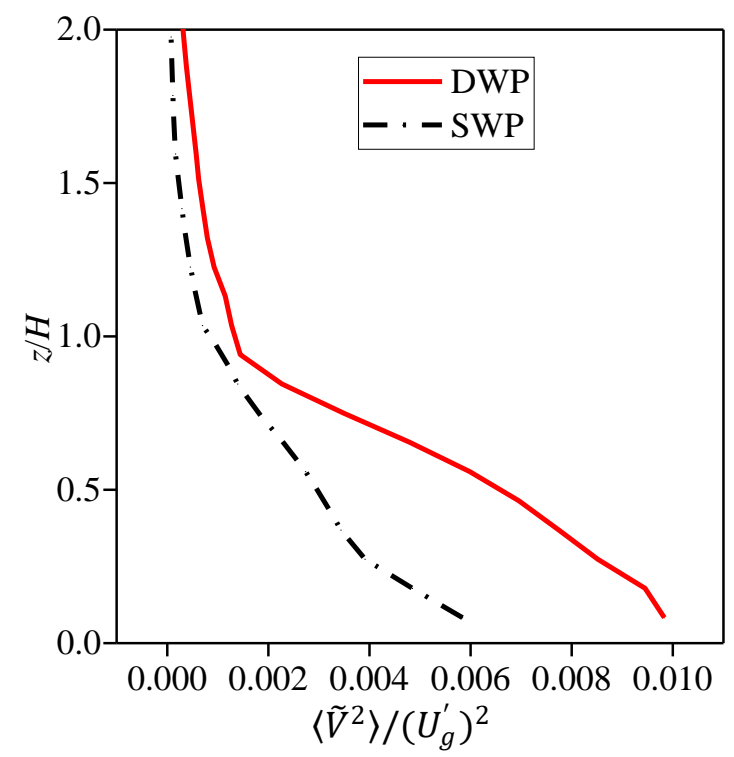

(c)

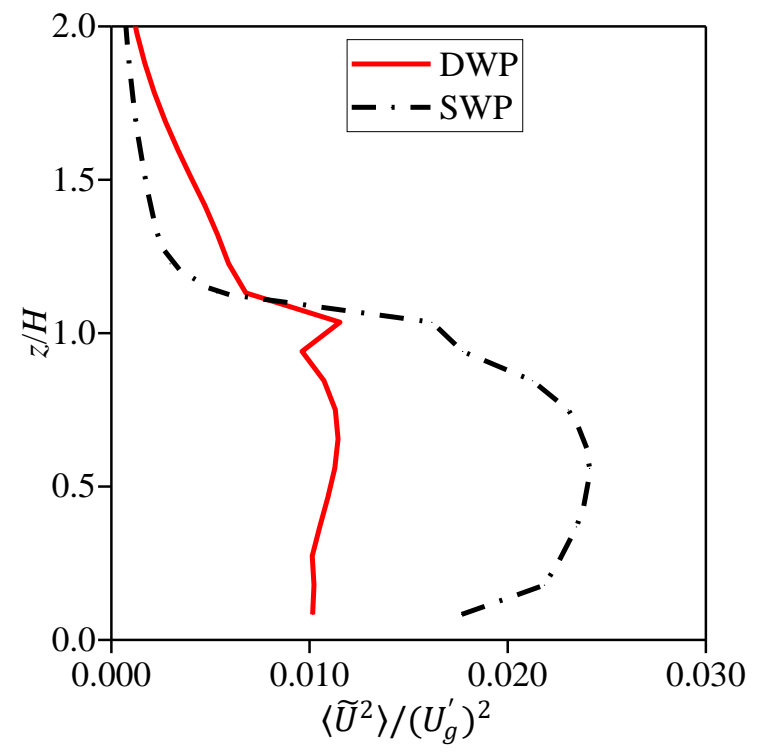

(b)

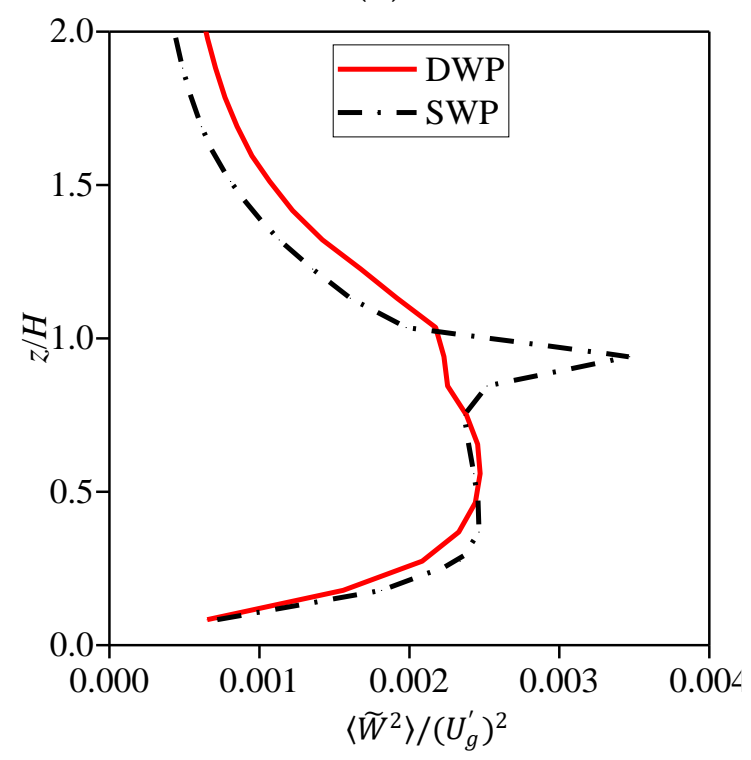

(d)

Figure 17. Dispersive stress components (a) $-\langle\widetilde{U} \widetilde{W}\rangle,(\mathbf{b})\left\langle\widetilde{U}^{2}\right\rangle,(\mathbf{c})\left\langle\widetilde{V}^{2}\right\rangle,(\mathbf{d})\left\langle\widetilde{W}^{2}\right\rangle$, normalized by $\left(U_{g}^{\prime}\right)^{2}$.

Figure 18 shows the profiles of kinetic energy, namely mean kinetic energy $\left(\mathrm{MKE}=0.5\left(U^{2}+V^{2}+W^{2}\right)\right.$, dispersive kinetic energy $\left(\mathrm{DKE}=0.5\left(\left\langle\widetilde{U}^{2}\right\rangle+\left\langle\widetilde{V}^{2}\right\rangle+\left\langle\widetilde{W}^{2}\right\rangle\right)\right)$, turbulent kinetic energy $\left(\mathrm{TKE}=0.5\left(\left\langle\bar{U}^{2}\right\rangle+\left\langle\bar{V}^{2}\right\rangle+\left\langle\bar{W}^{2}\right\rangle\right)\right)$, and total kinetic energy (TOTKE), which is the sum of the above kinetic energies, i.e., TOTKE = MKE + DKE + TKE. MKE dominates above the canopy. Within the urban canopy, the fraction of DKE increases, TKE is nearly uniform below $z / H<0.94$ for both cases, and DKE is comparable to TKE. The DKE for SWP exceeds TKE below $z / H<0.94$, which indicates the spatial inhomogeneity of time-averaged velocity caused by SWP. 


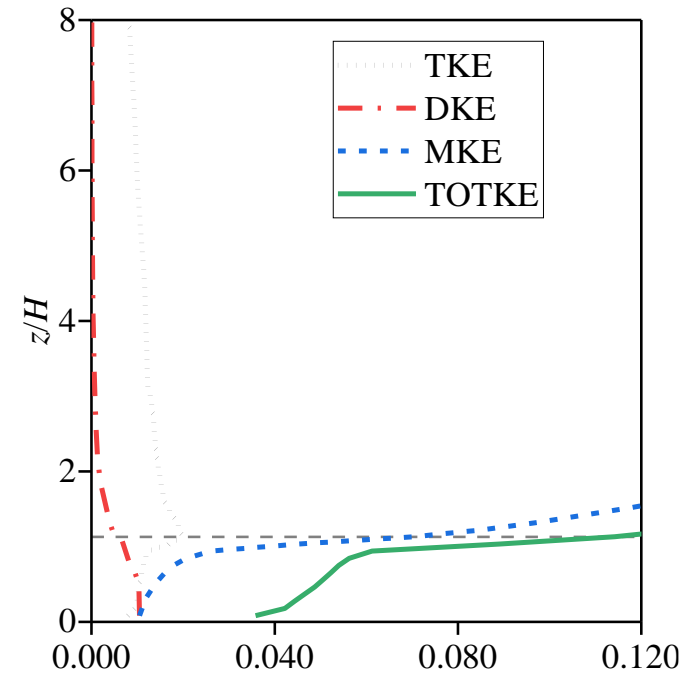

(a)

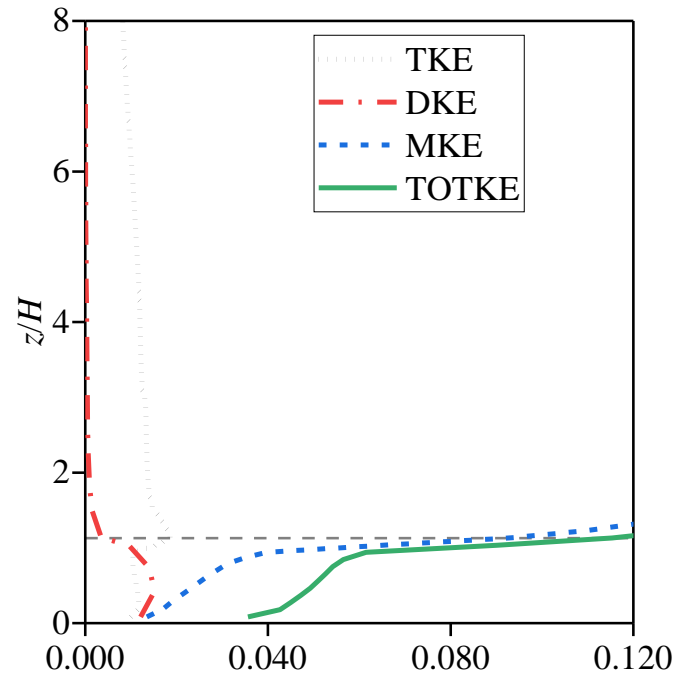

(b)

Figure 18. Profiles of TKE, DKE, MKE, and TOTKE for cases of (a) distorted wind passages DWP and (b) streamlined wind passages SWP.

\subsection{Drag Characteristics of Building Array}

\subsubsection{Drag Force Coefficients of the Local Buildings}

Figure 19a,b display the drag distributions of all individual buildings for both cases. The $\mathrm{C}_{\mathrm{d} 0}$ of each column displays similar distribution trends. Being exposed to the incoming flow directly, the $C_{d 0}$ of $\mathrm{R} 1$ are largest and roughly stabilize after the rapid drop from $R_{2}$, then fluctuates between 0 and 0.12 . The buildings behind $R_{1}$ are strongly sheltered by their preceding buildings and are exposed to lower velocities than the approaching flow, and therefore their drag forces are reduced.

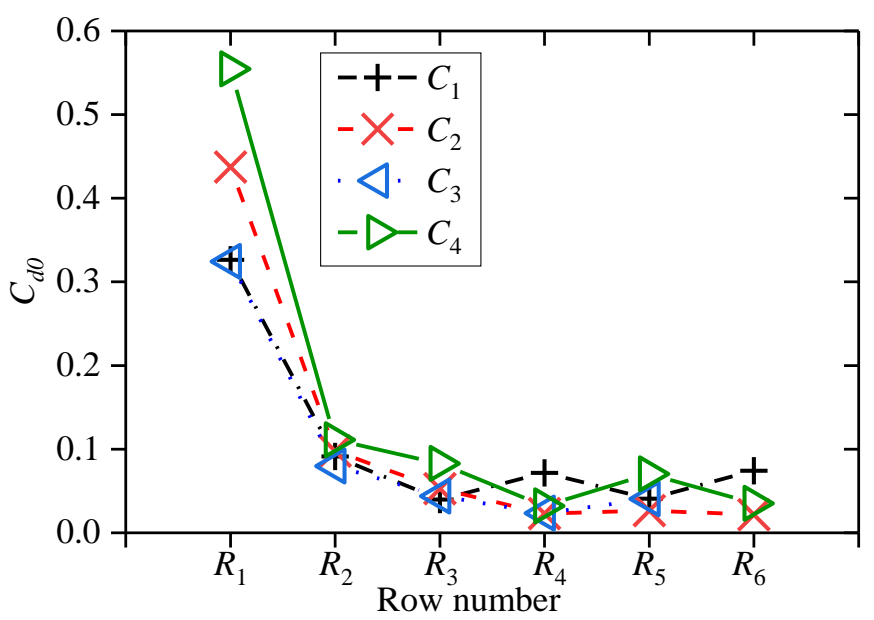

(a)

Figure 19. Cont. 


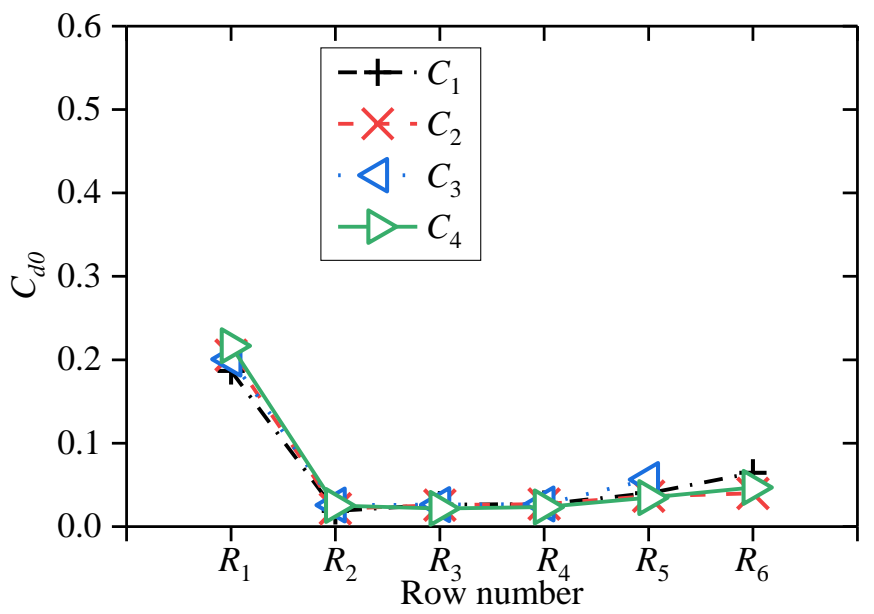

(b)

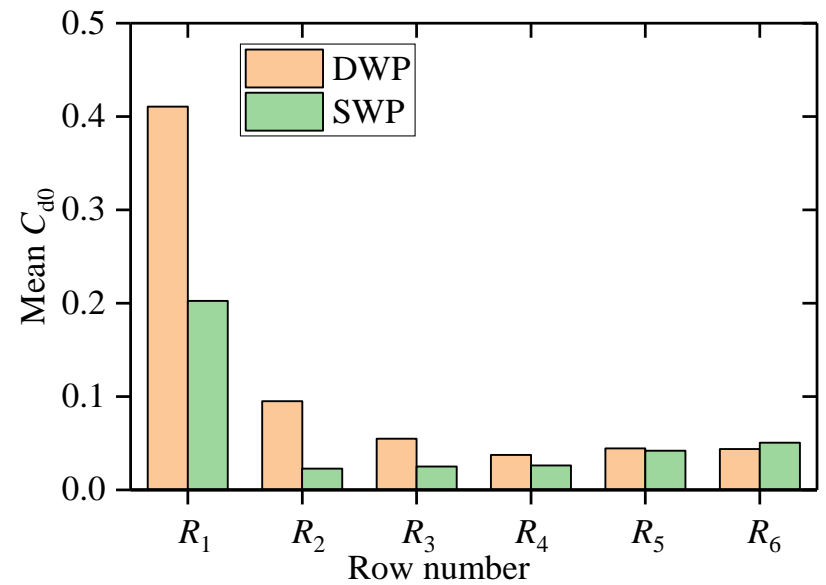

(c)

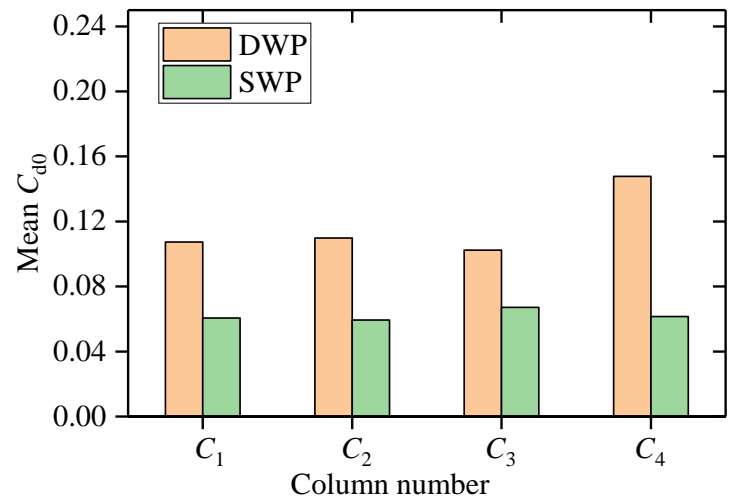

(d)

Figure 19. LES results of $C_{d 0}$ for (a) DWP, (b) SWP, (c) mean $C_{d 0}$ for all the individual buildings in each row, and (d) mean $C_{d 0}$ for all the individual buildings in each column.

Figure $19 \mathrm{c}$ depicts the mean $\mathrm{C}_{\mathrm{d} 0}$ for all the individual buildings in each row. For DWP, the $C_{\mathrm{d} 0}$ of $R_{1}$ accounts for $61 \%$ of the total drag force, followed by $14 \%$ of $R_{2}$, while for SWP, the above proportions are $56 \%$ of $R_{1}, 12 \%$ of $R_{5}, 11 \%$ of $R_{6}$, respectively. The total drag of DWP is stronger than the total drag of SWP. The $C_{\mathrm{d} 0}$ values of DWP far exceed those of SWP for the first two rows and are comparable to $R_{3}$ where the flow balance is reached. The mean $C_{\mathrm{d} 0}$ of DWP for $R_{1}$ exceeds $100 \%$ of SWP because the span-wise airflow 
speeds up the wake region and leads to more significant pressures on the front face of the buildings.

Figure 20 shows the standard deviation (STD) of $C_{d 0}$ of all the individual buildings in each row and each column, representing the span-wise and stream-wise inhomogeneity of $C_{d 0}$. In total, the STDs for each row and column in DWP are more significant than SWP, with a maximum of 12.7 and 2.6 times of SWP, respectively. The STDs of $C_{d 0}$ for DWP display the most considerable fluctuation of 0.109 for $R_{1}$, followed by $R_{6}$ and $R_{4}$ with STDs of 0.027 and $0.023 . \mathrm{C}_{\mathrm{d} 0}$ indicates the lowest fluctuation of 0.012 at $R_{2}$ because of the strong sheltering effect. As shown in Figure 20b, the STDs of $C_{4}$ for DWP have the maximum STD of 0.202 , followed by $C_{2}$ and $C_{3}$. For SWP, the STDs are almost the same and much smaller than those of DWP.

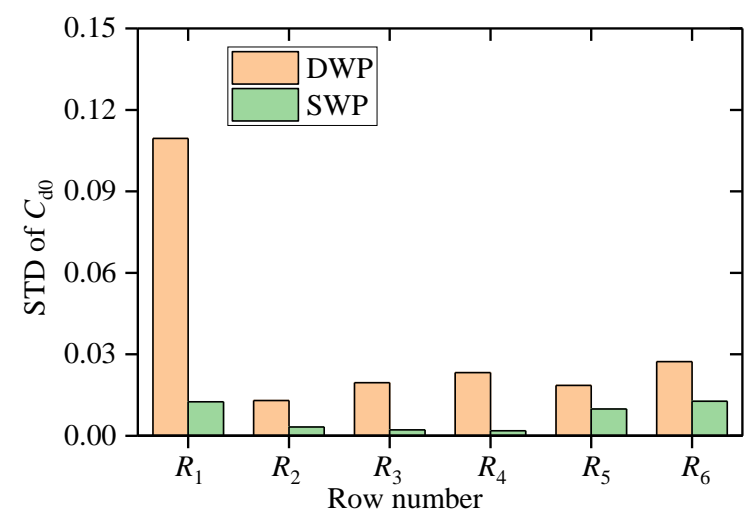

(a)

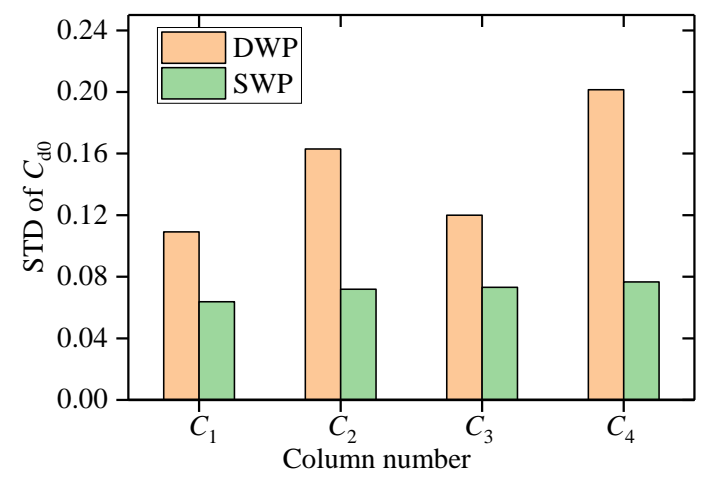

(b)

Figure 20. Comparisons of STD between DWP and SWP of $C_{d 0}(\mathbf{a})$ for each row and (b) for each column.

\subsubsection{Sectional Drag Force Coefficients of Local Buildings}

To further investigate the drag distributions along the vertical direction, this study introduces the sectional drag force coefficient $C_{d 0}^{z}$ for the building model, which is defined as [55]:

$$
C_{d 0}^{z}=\frac{1}{H} \frac{d C_{d 0}}{d z}
$$

Figure 21 shows the LES results of $C_{d 0}^{z}$ for six pressure modules $C_{2}$ along the wind direction for both cases. All $C_{d 0}^{z}$ curves decrease first, reaching the minimal local value at about $\mathrm{z}=0.2 \mathrm{H}$, then linearly increase with height until reaching the peak value around $z=0.9 \mathrm{H}$, and decay rapidly at the roof of the building. The $C_{d 0}^{z}$ profiles of SWP from $B_{2,2}$ converge, while for DWP, the profiles converge from $B_{2,3}$. The $C_{d 0}^{z}$ for DWP are larger than for SWP as a whole, again because of the span-wise airflow. The DNS results for the staggered array with equal frontal area density $\lambda_{p}$ but lower frontal area density $\lambda_{\mathrm{f}}$ of 0.25 than SWP are included in Figure 21a. The $C_{d 0}^{z}$ trend for the most windward building $B_{1,2}$ is similar to the DNS result, including the heights corresponding to the maximal and minimal 
$C_{d 0}^{z}$ values. $C_{d 0}^{z}$ of $B_{1,2}$ is larger than DNS when $z / H$ is below 0.8 , because $B_{1,2}$ is directly exposed to the incoming flow, without being sheltered by its preceding buildings, unlike in the DNS case. For other buildings, their $C_{d 0}^{z}$ are one order of magnitude smaller than the DNS case, because larger $\lambda_{\mathrm{f}}$ of SWP enables a stronger mutual sheltering effect than the DNS case.

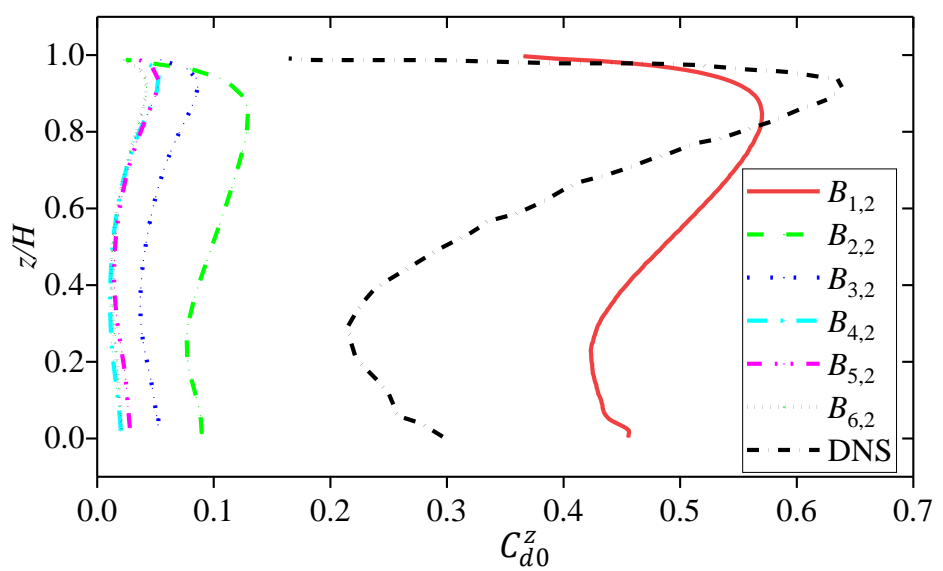

(a)

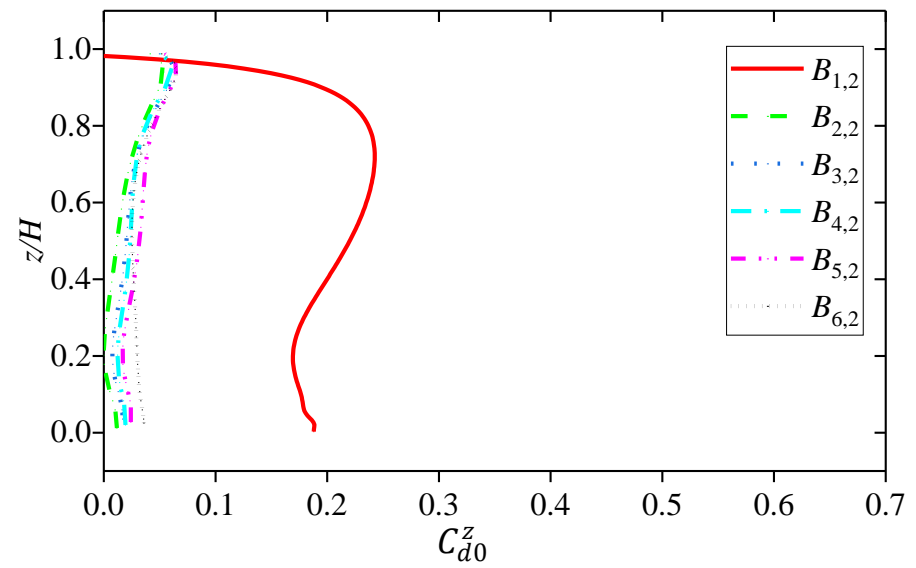

(b)

Figure 21. Sectional drag force coefficient $C_{d 0}^{z}$ for six pressure modules $C_{2}$ along wind direction for (a) DWP and (b) SWP. The DNS results are from the research of Santiago et al. [47].

Figure 22 further shows the heights $H_{\mathrm{p}}$, which correspond to the peak sectional drag coefficients for the two cases. $H_{\mathrm{p}}$ of $R_{1}$ and $R_{2}$ in DWP are more discrete than those of $R_{1}$ in SWP. They increase to $0.89 \mathrm{H}$ and stabilize from $R_{3}$, which is lower than $0.92 \mathrm{H}$ in the streamlined case. For DWP, the approaching flow can penetrate $R_{3}$ along the wind direction, and then a local flow balance is established from $C_{3}$ onward. This can also be inferred from the stable drag force after $R_{3}$ in Figure 19a. The flow adjustment distance of three rows is shorter than the two rows in SWP due to the intense flow mixing caused by distorted wind passages. $H_{\mathrm{p}}$ values fluctuate more significantly for $C_{1}, C_{2}$, and $C_{4}$ for DWP than for SWP. $H_{\mathrm{p}}$ of $C_{3}$ remains unchanged after $R_{3}$ for both cases, and we can infer that the skipping flow dominates the flow regime in the flow balance region. For DWP, $H_{\mathrm{p}}$ of $C_{2}$ remains unchanged at 0.92 at $R_{4}$ and $R_{5}$ and then decreases to 0.89 at $R_{6}$. For SWP, $H_{\mathrm{p}}$ of $C_{2}$ remains unchanged at 0.92 . This is because the distorted wind passage causes the recovery of the skipping flow, and it may reattach to the ground, and therefore airflow move down further into the urban canopy. The fluctuation of $H_{\mathrm{p}}$ for $C_{4}$ is largest in DWP, suggesting that the flow over $C_{4}$ interferes with its adjacent wind passages, verified by the most significant drag force for $C_{4}$. 


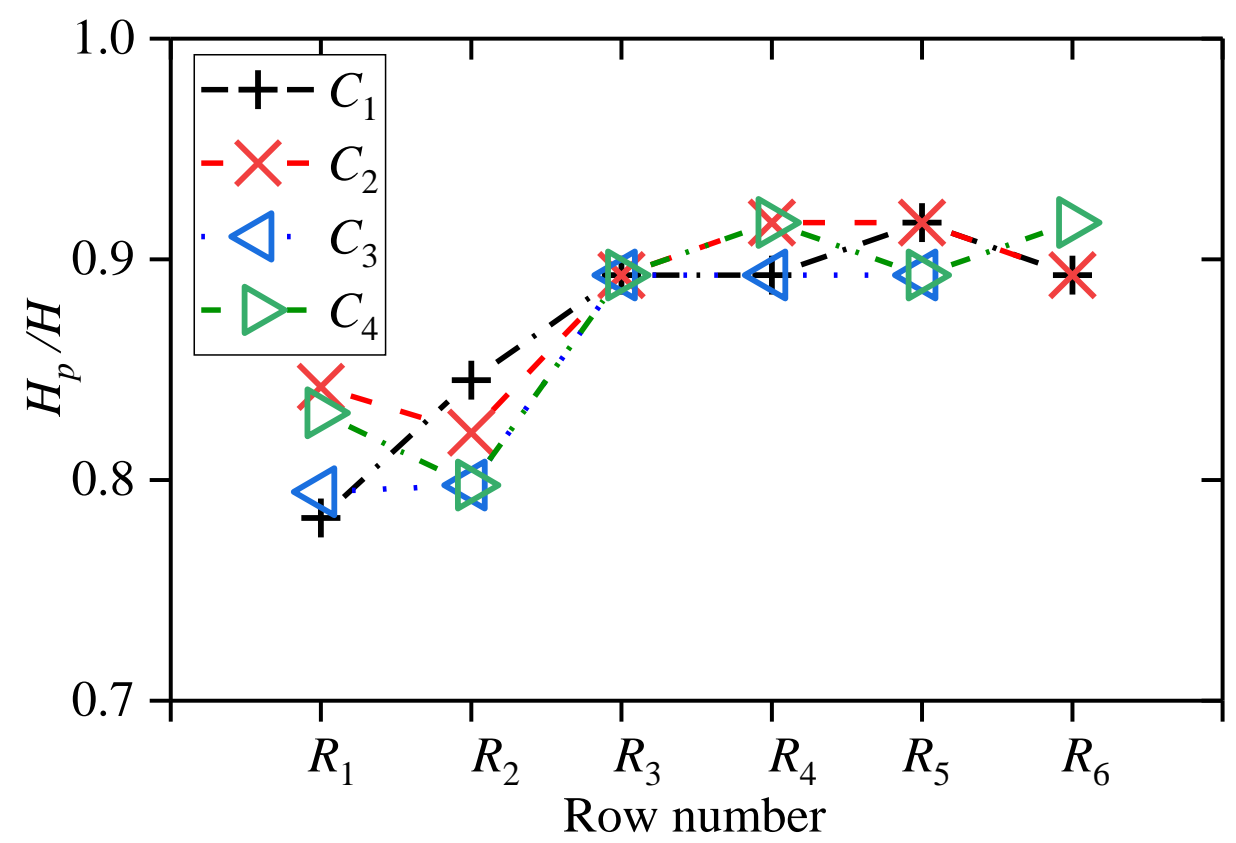

(a)

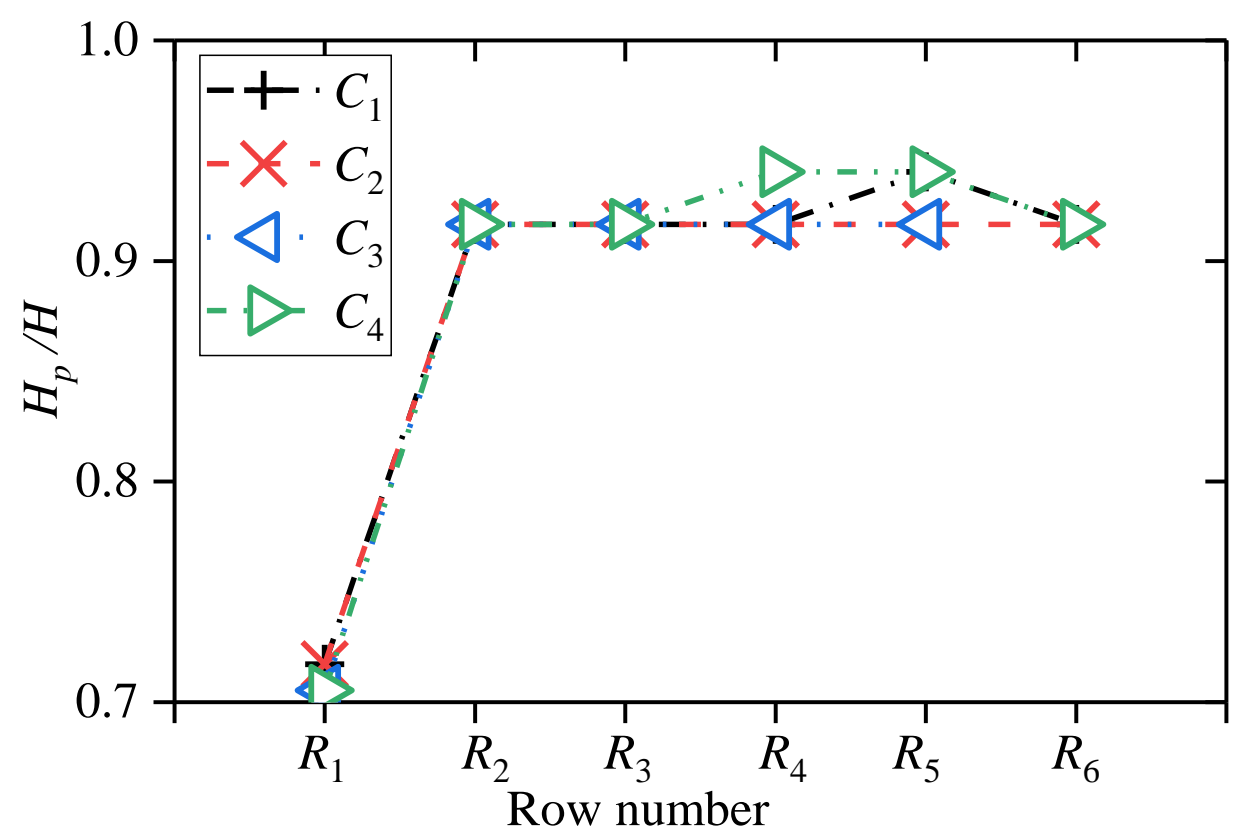

(b)

Figure 22. Heights corresponding to the peak sectional drag coefficients $H_{\mathrm{p}}$ normalized by the building height $H$ for (a) DWP and (b) SWP.

\section{Conclusions}

We summarized two typical types of wind passages for the complex buildings in real cities, i.e., the distorted and streamlined types, according to whether the central axis of each local wind passage is collinear or not. In this study, large-eddy simulations were conducted to explore the impacts of wind passages types on the wind and drag characteristics. Cases of distorted and streamlined wind passages (DWP and SWP) were designed. The recycling method by Kataoka and Mizuno [40] was applied to generate turbulent inflow data for LESs. The prediction accuracy of LES for estimating flow characteristics was evaluated 
by comparing it with the wind tunnel experiment results. Mean and unsteady flow characteristics within and above the canopy layer and drag force coefficients of six-row building models were assessed. The conclusions of this work can be summarized as follows:

(1) (Given the proper arrangement of the roughness models in the driver region, the inlet recycling method by Kataoka and Mizuno [40] can accurately reproduce the mean wind speeds, turbulence intensities, and stream-wise power spectral densities of the turbulent boundary layer. Moreover, the total drag forces of the building array $C_{d t}$ and drag forces of individual buildings $C_{d 0}$ coincide well with the experiment results.

(2) In the wake region, the streamlines tilt near the ground, and span-wise flow is detected at half the building height for DWP, while for SWP, the recirculating vortices dominate the wake region. The Reynolds stress and TKE profiles both occur at 1.13 times building height. The dispersive stress components $\left\langle\widetilde{V}^{2}\right\rangle$ and $\left\langle\widetilde{U}^{2}\right\rangle$ within the urban canopy layer for DWP are up to 2.5 and 1.7 times of those for SWP, indicating spatial inhomogeneity of time-averaged velocities for SWP.

(3) The individual drag forces $C_{d 0}$ for DWP are up to $100 \%$ larger than SWP for the preceding two rows and are comparable from the third row. The maximum fluctuations of $C_{d 0}$ for DWP along span-wise and stream-wise directions are 12.7 and 2.6 times of those for SWP. The profiles of sectional drag coefficients $C_{d 0}^{z}$ for SWP converged from $B_{2,2}$, while for DWP, they converged from $B_{2,3}$. The flow adjustment distance of three rows for DWP is shorter than the two rows for SWP.

These findings present helpful information that may serve as a foundation for urban design and planning. Some conclusions of this study may be explored further:

(1) This study extracted two typical wind passages from actual urban buildings, including distorted and streamlined types. Although we consider that they can represent the characteristics of building arrays, they are still different from real ones, and there are more types of wind passages among buildings. We must study more complex scenarios in future work to arrive at more generalized conclusions.

(2) The buildings in the array are of six rows, four columns, and the same height. The height variability of buildings and the urban length are also influential factors in the turbulent flow and flow adjustment process, which should be investigated in future studies.

Author Contributions: Conceptualization, L.W. and D.S.; Funding acquisition, J.L.; Investigation, L.W.; Methodology, J.L.; Project administration, J.L.; Resources, C.J. and Y.X.; Software, L.W.; Supervision, J.L., B.L. and M.L.; Validation, L.W.; Writing—original draft, L.W.; Writing—review \& editing, J.L. All authors have read and agreed to the published version of the manuscript.

Funding: This research was funded by the National Natural Science Foundation of China 51878208 and 51708151, China Postdoctoral Science Foundation (2019T120274), the Fundamental Research Funds for the Central Universities (HIT.NSRIF.2020049) and is related to the Joint Usage/Research Application in Tokyo Polytechnic University Wind Engineering Research Center.

Conflicts of Interest: The authors declare no conflict of interest.

\section{References}

1. Liu, Y.; Li, Q.; Yang, L.; Mu, K.; Zhang, M.; Liu, J. Urban heat island effects of various urban morphologies under regional climate conditions. Sci. Total. Environ. 2020, 743, 140589. [CrossRef]

2. Oke, T.R. The energetic basis of the urban heat island. Q. J. R. Met. Soc. 1982, 108, 1-24. [CrossRef]

3. Pakarnseree, R.; Chunkao, K.; Bualert, S. Physical characteristics of Bangkok and its urban heat island phenomenon. Build. Environ. 2018, 143, 561-569. [CrossRef]

4. Mishra, V.; Ganguly, A.R.; Nijssen, B.; Lettenmaier, D.P. Changes in observed climate extremes in global urban areas. Environ. Res. Lett. 2015, 10, 024005. [CrossRef]

5. Peng, L.; Liu, J.-P.; Wang, Y.; Chan, P.; Lee, T.; Peng, F.; Wong, M.; Li, Y. Wind weakening in a dense high-rise city due to over nearly five decades of urbanization. Build. Environ. 2018, 138, 207-220. [CrossRef]

6. Santamouris, M.; Papanikolaou, N.; Livada, I.; Koronakis, I.; Georgakis, C.; Argiriou, A.; Assimakopoulos, D.N. On the impact of urban climate on the energy consumption of buildings. Sol. Energy 2001, 70, 201-216. [CrossRef] 
7. Fan, Y.; Wang, Q.; Yin, S.; Li, Y. Effect of city shape on urban wind patterns and convective heat transfer in calm and stable background conditions. Build. Environ. 2019, 162, 106288. [CrossRef]

8. Lo, K.W.; Ngan, K. Characterising the pollutant ventilation characteristics of street canyons using the tracer age and age spectrum. Atmos. Environ. 2015, 122, 611-621. [CrossRef]

9. Blocken, B.; Carmeliet, J.; Stathopoulos, T. CFD evaluation of wind speed conditions in passages between parallel buildingseffect of wall-function roughness modifications for the atmospheric boundary layer flow. J. Wind. Eng. Ind. Aerodyn. 2007, 95, 941-962. [CrossRef]

10. Blocken, B.; Stathopoulos, T.; Carmeliet, J. Wind Environmental Conditions in Passages between Two Long Narrow Perpendicular Buildings. J. Aerosp. Eng. 2008, 21, 280-287. [CrossRef]

11. Li, B.; Luo, Z.; Sandberg, M.; Liu, J. Revisiting the 'Venturi effect' in passage ventilation between two non-parallel buildings. Build. Environ. 2015, 94, 714-722. [CrossRef]

12. Tse, K.T.; Weerasuriya, A.U.; Zhang, X.; Li, S.W.; Kwok, K.C.S. Effects of twisted wind flows on wind conditions in passages between buildings. J. Wind. Eng. Ind. Aerodyn. 2017, 167, 87-100. [CrossRef]

13. Allegrini, J.; Lopez, B. The influence of angular configuration of two buildings on the local wind climate. J. Wind. Eng. Ind. Aerodyn. 2016, 156, 50-61. [CrossRef]

14. Jamińska-Gadomska, P.; Lipecki, T.; Pieńko, M.; Podgórski, J. Wind velocity changes along the passage between two angled walls-CFD simulations and full-scale measurements. Build. Environ. 2019, 157, 391-401. [CrossRef]

15. Yoshida, T.; Takemi, T.; Horiguchi, M. Large-Eddy-Simulation Study of the Effects of Building-Height Variability on Turbulent Flows over an Actual Urban Area. Bound.-Layer Meteorol. 2018, 168, 127-153. [CrossRef]

16. Antoniou, N.; Montazeri, H.; Wigo, H.; Neophytou, M.K.-A.; Blocken, B.; Sandberg, M. CFD and wind-tunnel analysis of outdoor ventilation in a real compact heterogeneous urban area: Evaluation using "air delay". Build. Environ. 2017, 126, 355-372. [CrossRef]

17. Cheng, H.; Hayden, P.; Robins, A.G.; Castro, I.P. Flow over cube arrays of different packing densities. J. Wind. Eng. Ind. Aerodyn. 2007, 95, 715-740. [CrossRef]

18. Coceal, O.; Thomas, T.G.; Castro, I.P.; Belcher, S.E. Mean Flow and Turbulence Statistics over Groups of Urban-like Cubical Obstacles. Bound.-Layer Meteorol. 2006, 121, 491-519. [CrossRef]

19. Hagishima, A.; Tanimoto, J.; Nagayama, K.; Meno, S. Aerodynamic Parameters of Regular Arrays of Rectangular Blocks with Various Geometries. Bound.-Layer Meteorol. 2009, 132, 315-337. [CrossRef]

20. Zaki, S.A.; Hagishima, A.; Tanimoto, J.; Ikegaya, N. Aerodynamic Parameters of Urban Building Arrays with Random Geometries. Bound.-Layer Meteorol. 2011, 138, 99-120. [CrossRef]

21. $\mathrm{Hu}, \mathrm{T}$; Y Yoshie, R. Indices to evaluate ventilation efficiency in newly-built urban area at pedestrian level. J. Wind. Eng. Ind. Aerodyn. 2013, 112, 39-51. [CrossRef]

22. Lin, M.; Hang, J.; Li, Y.; Luo, Z.; Sandberg, M. Quantitative ventilation assessments of idealized urban canopy layers with various urban layouts and the same building packing density. Build. Environ. 2014, 79, 152-167. [CrossRef]

23. Chen, G.; Rong, L.; Zhang, G. Impacts of urban geometry on outdoor ventilation within idealized building arrays under unsteady diurnal cycles in summer. Build. Environ. 2021, 206, 108344. [CrossRef]

24. Hanna, S.R.; Tehranian, S.; Carissimo, B.; Macdonald, R.W.; Lohner, R. Comparisons of model simulations with observations of mean flow and turbulence within simple obstacle arrays. Atmos. Environ. 2002, 36, 5067-5079. [CrossRef]

25. Kono, T.; Tamura, T.; Ashie, Y. Numerical Investigations of Mean Winds within Canopies of Regularly Arrayed Cubical Buildings Under Neutral Stability Conditions. Bound.-Layer Meteorol. 2010, 134, 131-155. [CrossRef]

26. Maronga, B.; Gryschka, M.; Heinze, R.; Hoffmann, F.; Kanani-Sühring, F.; Keck, M.; Ketelsen, K.; Letzel, M.O.; Sühring, M.; Raasch, S. The Parallelized Large-Eddy Simulation Model (PALM) version 4.0 for atmospheric and oceanic flows: Model formulation, recent developments, and future perspectives. Geosci. Model Dev. Discuss. 2015, 8, 1539-1637. [CrossRef]

27. Inagaki, A.; Castillo, M.C.L.; Yamashita, Y.; Kanda, M.; Takimoto, H. Large-Eddy Simulation of Coherent Flow Structures within a Cubical Canopy. Bound.-Layer Meteorol. 2012, 142, 207-222. [CrossRef]

28. Kanda, M.; Moriwaki, R.; Kasamatsu, F. Large-Eddy Simulation of Turbulent Organized Structures within and above Explicitly Resolved Cube Arrays. Bound.-Layer Meteorol. 2004, 112, 343-368. [CrossRef]

29. Razak, A.A.; Hagishima, A.; Ikegaya, N.; Tanimoto, J. Analysis of airflow over building arrays for assessment of urban wind environment. Build. Environ. 2013, 59, 56-65. [CrossRef]

30. Ikegaya, N.; Ikeda, Y.; Hagishima, A.; Tanimoto, J. Evaluation of rare velocity at a pedestrian level due to turbulence in a neutrally stable shear flow over simplified urban arrays. J. Wind. Eng. Ind. Aerodyn. 2017, 171, 137-147. [CrossRef]

31. Biehl, J.; Paas, B.; Klemm, O. Ventilation of a Mid-Size City under Stable Boundary Layer Conditions: A Simulation Using the LES Model PALM. Atmosphere 2021, 12, 401. [CrossRef]

32. Gronemeier, T.; Raasch, S.; Ng, E. Effects of Unstable Stratification on Ventilation in Hong Kong. Atmosphere 2017, 8, 168. [CrossRef]

33. Park, S.-B.; Baik, J.-J.; Ryu, Y.-H. A Large-Eddy Simulation Study of Bottom-Heating Effects on Scalar Dispersion in and above a Cubical Building Array. J. Appl. Meteorol. Climatol. 2013, 52, 1738-1752. [CrossRef]

34. Kurppa, M.; Hellsten, A.; Auvinen, M.; Raasch, S.; Vesala, T.; Järvi, L. Ventilation and Air Quality in City Blocks Using Large-Eddy Simulation-Urban Planning Perspective. Atmosphere 2018, 9, 65. [CrossRef] 
35. Deardorff, J.W. Stratocumulus-capped mixed layers derived from a three-dimensional model. Bound.-Layer Meteorol. 1980, 18, 495-527. [CrossRef]

36. Miller, M.J.; Thorpe, A.J. Radiation conditions for the lateral boundaries of limited-area numerical models: Radiation boundary conditions for numerical models. Q. J. R. Meteorol. Soc. 1981, 107, 615-628. [CrossRef]

37. Khanna, S.; Brasseur, J.G. Analysis of Monin-Obukhov similarity from large-eddy simulation. J. Fluid Mech. 1997, 345, 251-286. [CrossRef]

38. Zhang, J. Fast and High Accuracy Multigrid Solution of the Three Dimensional Poisson Equation. J. Comput. Phys. 1998, 143, 449-461. [CrossRef]

39. Tabor, G.R.; Baba-Ahmadi, M.H. Inlet conditions for large eddy simulation: A review. Comput. Fluids 2010, 39, 553-567. [CrossRef]

40. Kataoka, H.; Mizuno, M. Numerical flow computation around aeroelastic 3D square cylinder using inflow turbulence. Wind. Struct. 2002, 5, 379-392. [CrossRef]

41. Nozawa, K.; Tamura, T. Large eddy simulation of the flow around a low-rise building immersed in a rough-wall turbulent boundary layer. J. Wind. Eng. Ind. Aerodyn. 2002, 90, 1151-1162. [CrossRef]

42. Li, B.; Cunyan, J.; Lu, W.; Weihua, C.; Jing, L. A parametric study of the effect of building layout on wind flow over an urban area. Build. Environ. 2019, 160, 106160. [CrossRef]

43. Tominaga, Y.; Mochida, A.; Yoshie, R.; Kataoka, H.; Nozu, T.; Yoshikawa, M.; Shirasawa, T. AIJ guidelines for practical applications of CFD to pedestrian wind environment around buildings. J. Wind. Eng. Ind. Aerodyn. 2008, 96, 1749-1761. [CrossRef]

44. Xie, Z.-T.; Coceal, O.; Castro, I.P. Large-Eddy Simulation of Flows over Random Urban-like Obstacles. Bound.-Layer Meteorol. 2008, 129, 1-23. [CrossRef]

45. Yang, X.I.A.; Xu, H.H.A.; Huang, X.L.D.; Ge, M.-W. Drag forces on sparsely packed cube arrays. J. Fluid Mech. 2019, 880, 992-1019. [CrossRef]

46. Liu, J.; Niu, J.; Du, Y.; Mak, C.M.; Zhang, Y. LES for pedestrian level wind around an idealized building array-Assessment of sensitivity to influencing parameters. Sustain. Cities Soc. 2019, 44, 406-415. [CrossRef]

47. Bou-Zeid, E.; Overney, J.; Rogers, B.D.; Parlange, M.B. The Effects of Building Representation and Clustering in Large-Eddy Simulations of Flows in Urban Canopies. Bound.-Layer Meteorol. 2009, 132, 415-436. [CrossRef]

48. Hanna, S.R.; Hansen, O.R.; Dharmavaram, S. FLACS CFD air quality model performance evaluation with Kit Fox, MUST, Prairie Grass, and EMU observations. Atmos. Environ. 2004, 38, 4675-4687. [CrossRef]

49. Moonen, P.; Allegrini, J. Employing statistical model emulation as a surrogate for CFD. Environ. Model. Softw. 2015, 72, 77-91. [CrossRef]

50. Schatzmann, M.; Olesen, H.; Franke, J. Cost 732 Model Evaluation Case Studies: Approach and Results; COST Office: Hamburg, Germany, 2010; p. 123.

51. Castro, I.P.; Cheng, H.; Reynolds, R. Turbulence over Urban-type Roughness: Deductions from Wind-tunnel Measurements. Bound.-Layer Meteorol. 2006, 118, 109-131. [CrossRef]

52. Cheng, H.; Castro, I.P. Near Wall Flow over Urban-like Roughness. Bound.-Layer Meteorol. 2002, 104, 229-259. [CrossRef]

53. Li, B.; Wang, L.; Liu, Y.; Liu, J. Drag distributions of non-uniform buildings from surface pressure measurements in wind tunnel. Build. Environ. 2018, 143, 618-631. [CrossRef]

54. Slotnick, J.; Khodadoust, A.; Alonso, J.; Darmofal, D.; Gropp, W.; Lurie, E.; Mavriplis, D. CFD Vision 2030 Study: A Path to Revolutionary Computational Aerosciences; NASA: Wanshington, DC, USA, 2014; p. 58.

55. Coceal, O.; Belcher, S.E. A canopy model of mean winds through urban areas. Q. J. R. Meteorol. Soc. 2004, 130, 1349-1372. [CrossRef] 